\title{
A review of Conocetus Desbrochers des Loges, 1875, subgenus of Polydrusus Germar, 1817 (Coleoptera, Curculionidae, Entiminae)
}

\author{
Christoph Germann \\ Naturmuseum Solothurn, Klosterplatz 2, CH-4500 Solothurn and Naturhistorisches Museum der \\ Burgergemeinde Bern, Bernastrasse 15, CH-3005 Bern, Switzerland. \\ Email: germann.christoph@gmail.com
}

urn:lsid:zoobank.org:author:0FFE5732-6AE7-4857-B837-54D30F41F573

\begin{abstract}
The species of the subgenus Conocetus Desbrochers des Loges, 1875 are reviewed and Polydrusus (Conocetus) transjordanus sp. nov. is described. Upon examination of the holotype of Polydrusus bardus Gyllenhal, 1834, it was observed that the species hitherto determined sensu auctorum as $P$. bardus was a misidentification. The specimen in question was therefore unnamed and is thus newly described as Polydrusus (Conocetus) crinipes sp. nov. Polydrusus femoratus (Stierlin, 1888 ) is a junior synonym of $P$. angustus (Lucas, 1854). Polydrusus gracilicornis Kiesenwetter, 1864, P. cylindrithorax (Desbrochers des Loges, 1900) and P. quadraticollis (Desbrochers des Loges, 1902) are proposed as junior synonyms of P. bardus. Polydrusus zurcheri (Schilsky, 1912) is proposed as a junior synonym of $P$. grandiceps (Desbrochers des Loges, 1875). Polydrusus kahri Kirsch, 1865 is transferred from subgenus Conocetus to Denticonocetus subgen. nov., with P. siculus Desbrochers des Loges, 1872 and $P$. vodozi Desbrochers des Loges, 1903 both recognized as new junior synonyms of P. kahri. The lectotypes of P. gracilicornis, P. zurcheri, P. marcidus Kiesenwetter, 1864, P. gracilis (Stierlin, 1888), P. rhodiacus (Schilsky, 1912) and P. grandiceps are designated. A key, figures, label data and distribution maps are provided for all species, except for P. longus (Stierlin, 1884), for which no specimens were available for examination, and whose placement in the subgenus Conocetus remains uncertain (thus categorized as incertae sedis). Polydrusus angustus is recorded for the first time for Italy, $P$. rhodiacus for mainland Turkey and P. festae (Solari, 1925) for Greece.
\end{abstract}

Keywords. Polydrusini, Polydrusus, Conocetus, Denticonocetus, new subgenus, new species, new synonyms, new records, taxonomy, Eastern Mediterranean region.

Germann C. 2018. A review of Conocetus Desbrochers des Loges, 1875, subgenus of Polydrusus Germar, 1817 (Coleoptera, Curculionidae, Entiminae). European Journal of Taxonomy 392: 1-39.

https://doi.org/10.5852/ejt.2018.392

\section{Introduction}

Desbrochers des Loges (1875) established the genus Conocetus for three species: Polydrusus bardus Gyllenhal, 1834, P. virens (Kiesenwetter, 1864) and the type species Conocetus grandiceps Desbrochers des Loges, 1875. He later provided a first review of the genus (Desbrochers des Loges 1902), including a determination key, new descriptions and redescriptions of several species. 
Schilsky (1910) included Conocetus as a subgenus in Polydrusus Germar, 1817 based on the similarity of the head and rostrum with the subgenera Leucodrosus Seidlitz, 1890 (type species P. tibialis Gyllenhal, 1834), Tylodrusus Stierlin, 1884 (type species P. pterygomalis Boheman, 1840) and Eustolus C.G. Thomson, 1859 (at present a senior synonym of Tylodrusus). This proposition was followed by all subsequent authors.

The most recently described new species in the subgenus Conocetus is $P$. (Conocetus) festae (Solari, 1925), based on specimens from Libya (Solari 1925).

The subgenus Conocetus is characterized as follows: 1) rostrum broad, transverse; 2) antennal scrobes lateral, short and triangular with lower margin protruding downwards and obtusely angled; 3) left mandible stronger, rectangularly protruding; 4) temples always well visible; 5) eyes oval to long-oval, more or less bulged, protruding from the head's outline; 6) antennae very slender and thin; 7) pronotum mostly as long as wide; 8) legs slender, femora without teeth; 9) integument of elytra and pronotum with circular to oval adjacent scales and bare areas where hairs arise.

Yunakov (2013) listed 19 species of Conocetus in the Catalogue of Palaearctic Coleoptera and a further 18 are listed in the Cooperative Catalogue of the Palaearctic Coleoptera Curculionoidea (AlonsoZarazaga et al. 2017: 354-364), excluding the erroneous repetition of Polydrusus (Conocetus) virens (Kiesenwetter, 1864).

After the present review, Conocetus and Denticonocetus subgen. nov. consist of 12 species, all distributed in the Eastern Mediterranean region, except for P. longus (Stierlin, 1884) from Morocco - reported here as incertae sedis.

\section{Material and methods}

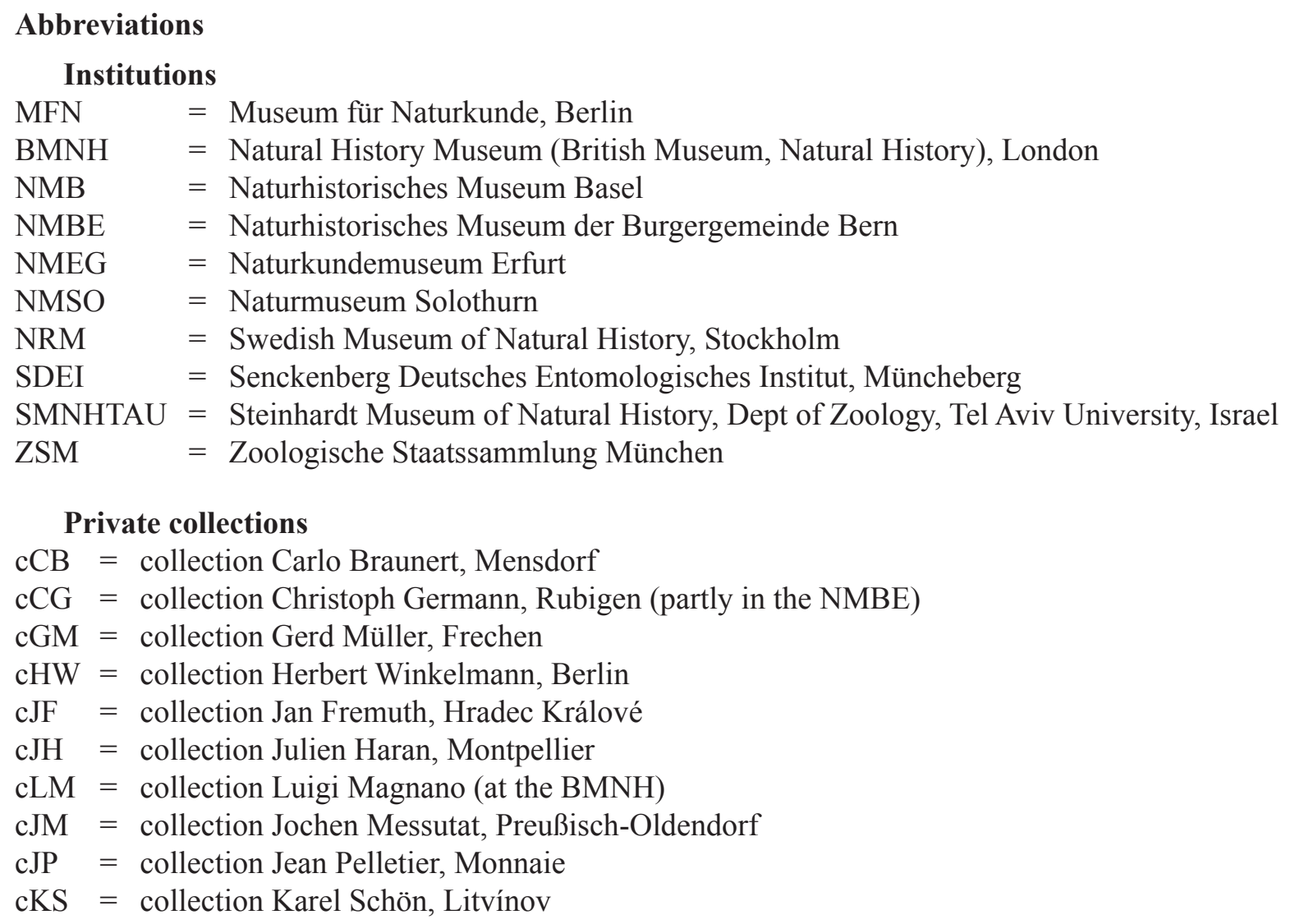


$\mathrm{cPB}=$ collection Piotr Białooki, Sopot

$\mathrm{cRB}=$ collection Roman Borovec, Sloupno

Collection localities are cited directly from labels with original spelling and punctuation. For type specimens, the different specimen labels are given from the top downward and separated by a double slash (//). Additional information and general remarks are highlighted using square brackets [ ].

Photographs were taken with a 5-megapixel digital camera (Leica DFC 420). Series of images were captured through a binocular microscope (Leica MZ16) and processed with Auto-Montage software (Imagic Image Access, Version 8). All measurements were taken digitally with the measurement-tool of the above mentioned Auto-Montage software. Body length was measured from the anterior margin of the eye to the apex of the elytra.

\section{Results}

Superfamily Curculionoidea Latreille, 1802

Family Curculionidae Latreille, 1802

Genus Polydrusus Germar, 1817

Subgenus Conocetus Desbrochers des Loges, 1875

Subgenus Denticonocetus subgen. nov.

Below is an overview of the presently valid species belonging to the subgenera Conocetus and Denticonocetus subgen. nov., as well as their synonyms as proposed here.

\section{Subgenus Conocetus Desbrochers des Loges, 1875}

Polydrusus (Conocetus) angustus (Lucas, 1854)

Eusomus angustus Lucas, 1854: 38

Eusomus angusticollis Lucas, 1854: 39

Conocetus virens Kiesenwetter, 1864: 252

Conocetus femoratus Stierlin, 1888a: 56 syn. nov.

Conocetus rotundicollis Desbrochers des Loges, 1902: 126

Polydrusus (Conocetus) bardus Gyllenhal, 1834

Polydrusus bardus Gyllenhal in Schoenherr et al., 1834: 143

Polydrusus gracilicornis Kiesenwetter, 1864: 253 syn. nov.

Conocetus graecus Stierlin, 1884: 81

Conocetus cylindrithorax Desbrochers des Loges, 1900: 14 syn. nov.

Conocetus quadraticollis Desbrochers des Loges, 1902: 129 syn. nov.

Conocetus quadricollis - Schilsky 1910: 22 (= lapsus)

Conocetus cylindricollis -Schilsky 1910: 23 (= homonym of Polydrusus (Leucodrusus) cylindricollis Boheman, 1840; lapsus)

Conocetus cylindrocollis - Yunakov 2013: 368 (= lapsus)

Polydrusus (Conocetus) baudii (Faust, 1889)

Conocetus baudii Faust, 1889: 73

Polydrusus (Conocetus) calabricus (Faust, 1890)

Conocetus calabricus Faust, 1890: 330 
Polydrusus (Conocetus) crinipes sp. nov. [=P. bardus sensu auctorum $]$

Polydrusus (Conocetus) festae (F. Solari, 1925)

Conocetus festae F. Solari, 1925: 29

Polydrusus (Conocetus) grandiceps (Desbrochers des Loges, 1875)

Conocetus grandiceps Desbrochers des Loges, 1875: 5

Conocetus zurcheri Schilsky, 1912: 18 syn. nov.

Polydrusus (Conocetus) marcidus Kiesenwetter, 1864

Polydrusus marcidus Kiesenwetter, 1864: 253

Conocetus gracilis Stierlin, 1888b: 14

Conocetus bardus Desbrochers des Loges, 1902: 124 (= homonym of Polydrusus (Conocetus) bardus Gyllenhal, 1834)

Conocetus dubius Schilsky, 1910: 21

Polydrusus (Conocetus) rhodiacus (Schilsky, 1912)

Conocetus rhodiacus Schilsky, 1912: 17

Polydrusus (Conocetus) stierlini (Schilsky, 1910)

Conocetus stierlini Schilsky, 1910: 24

Polydrusus (Conocetus) transjordanus sp. nov.

Denticonocetus subgen. nov.

Polydrusus (Denticonocetus) kahri Kirsch, 1865

Polydrusus kahri Kirsch, 1865: 122

Polydrusus gentilis Bertolini, 1868: 120

Eustolus florentinus Chevrolat, 1869: 73

Eustolus baldensis Stierlin, 1884: 69

Polydrusus siculus Desbrochers des Loges, 1872, p. 233 syn. nov.

Polydrusus vodozi Desbrochers des Loges, 1903: 114 syn. nov.

\section{Polydrusus incertae sedis}

Polydrusus longus (Stierlin, 1884)

Conocetus longus Stierlin, 1884: 80

Denticonocetus subgen. nov.

urn:1sid:zoobank.org:act:15B1 A726-F663-4A12-9AD8-DA0925B29BC2

\section{Type species}

Polydrusus kahri Kirsch, 1865

\section{Diagnosis}

A new subgenus of Polydrusus Germar, 1817, similar to Conocetus, especially in the broad rostrum and the shape of the antennal scrobes (see Introduction), but differing in: (1) all femora toothed (untoothed in Conocetus) and (2) internal sac consisting of a tissue with rasp-like teeth and a simple, clip-shaped sclerite as shown in Fig. 9A-C. In Conocetus the internal sac consists of a complex "wrench"-shaped 
sclerite, see Fig. 9D-P, whereas in Eustolus the internal sac consists of several differently shaped, complex sclerites, and in the rather heterogeneous Polydrusus s. str. the internal sacs consist of prevailing tissues with rasp-like teeth.

\section{Etymology}

The subgeneric name Denticonocetus subgen. nov. is of male gender and combines the characteristically dentate femora (in Latin: dentis = tooth) and the morphologically most similar subgenus Conocetus.

Polydrusus (Denticonocetus subgen. nov.) kahri Kirsch, 1865

Figs 1A-B, 7A, 9A-C, 10

\section{Material examined}

\section{Syntypes}

ITALY: 1 §, 1 क, kahri Kirsch A.la Mt Baldo Tyrol Kahr // 407 // Sch. // coll. L.v. Heyden DEI Müncheberg // [red label:] SYNTYPUS Polydrusus (Denticonocetus) kahri Kirsch, 1865 labelled C. Germann 2015 (SDEI).

\section{Type locality}

ITALY: Monte Baldo.

\section{Other material examined}

AUSTRIA: 3 ex., Teriolis [Zirl, Innsbruck], coll. Koltze (SDEI).

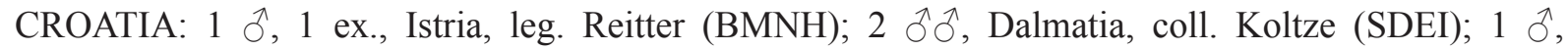
1 , , Istria, Krk Island, Baska env., leg. E. Strejckova (cRB); 2 ex., Istrien, Vrsar, 17 Jul. 1988, leg. W. Suppantschitsch (SDEI); 1 ex., Istrien, Dvigrad, 20 Jul. 1988, leg. W. Suppantschitsch (SDEI).

FRANCE: 1 ð, Corsica, Furiani, 10 Jul. 2015, flight interception trap, Quercus, leg. J. Haran (cJH).

GREECE: 1 + , Isl. Zakynthos, Limni Keriou, Villa Artemis, $37.681193^{\circ} \mathrm{N}, 20.829127^{\circ} \mathrm{E}, 37 \mathrm{~m}, 22$ May 2014, leg. U. Schneppat (cCG).

ITALY: 1 ex., Florence (cJP); 1 ex., Scala, 5 May [19]36, leg. F. Vitale (cJP); 1 ex., San Casciano Val di Pesa (FI), 4 Jul. 1963, leg. Giannini (BMNH); 1 ex., Ancona, leg. Flach (BMNH); 1 ex., San Casciano Val di Pesa, Jul. [19]88, leg. Giannini (BMNH); 1 ex., Abruzzo, leg. Fiori (BMNH); 3 ex. Sicilia (PA), $5 \mathrm{~km} \mathrm{SW}$ of Carini, Mt Longa, 38 $07^{\prime} 04^{\prime \prime} \mathrm{N}, 13^{\circ} 08^{\prime} 06^{\prime \prime} \mathrm{E}, 640 \mathrm{~m}$, Kalk, Quercus-Wald [forest], 27 Jul. 2005, leg. C. Germann (cCG); 1 गे, 2 ex., Sicilia (PA), 3 km N of Bisacquino, 37 $33^{\circ} 44^{\prime \prime}$ N, $13^{\circ} 16^{\prime} 05^{\prime \prime}$ E, 830 m, Kalk, Quercus ilex, 1 Aug. 2005, leg. Germann (cCG, NMBE); 1 ex., Sicilia (PA),

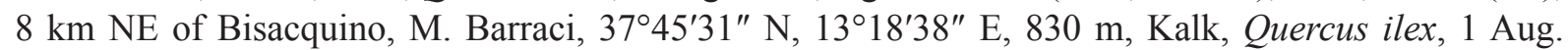
2005, leg. Germann (cCG); 1 ô, Sizilien, coll. Rätzer (NMBE); 1 ô, 1 ㅇ, Ancona, Mte. Conero, 200 m, 15 Jun.1991, leg. R. Borovec (cRB); 1 ex., Abruzzo (AQ), Gran Sasso, Ponte Cerreta, 1060 m, 24 Jun. 1991, leg. R. Borovec (cRB); 3 ex., Lazio (RI), Casaprota, env. Quercetum, 250 m, 22 Jun. 1991, leg. R. Borovec (cRB); 1 q, Sicilia, Mte Soro, 10 km NW of Cesarò, P.N. dei Nebrodi, 19 Oct. 2002, leg. C. Germann (NMBE); 3 ex., Abruzzo, Mt Aragno, 2 Aug. 1898, leg. A. Fiori, coll. A. Rätzer (NMBE); 1 ex., Sicile, Castelbuono, 16 May 2008, leg. Cantot (cJP); 2 ex., Sicilia, M. Nebrodi, S. Fratello, 14 Jun. 1996, leg. Krajcik (cRB); 1 ð̄, 1 ㅇ, Sicilia bor. Or. Francavilla di Sicilia, 400-600 m, 7-9 May 1995, leg. R. Borovec (cRB); 2 ex., Abruzzo, Mt Aragno, 2 Aug. 1898, leg. A. Fiori (NMB); 1 ex., Abruzzen, coll. J. Breit (NMB); 1 ex., Abruzzen, Mt Aragno, coll. J. Breit (NMB); 1 + , Sicilien, 1888, leg. Stierlin, coll. Apfelbeck (NMB); 1 ex., Emilia, coll. J. Breit (NMB); 2 ex., Trento, Scriba, coll. L.v. Heyden (SDEI); 2 ex., Tyrol, Kahr, coll. L.v. Heyden (SDEI); 2 ex., Gran Sasso, Sequens, coll. L.v. Heyden 
(SDEI); 5 ex., Sicilia, coll. L.v. Heyden (SDEI); 1 ex., Umbrien, Hochebene von Castelluccio, 1400 m, Aug. 1997, leg. M. Langer (SDEI); 1 J , Campania, Monti Alburni, 600 m, 10 Jul. 1996, San Lorenzo, leg. W. Suppantschitsch (SDEI); 1 đ̃, Palermo, Bosco della Ficuzza, 1000 m, 10 Jul. 2001, leg. W. Suppantschitsch (SDEI); 1 , Sicilia (PA), 8 km N of Petralis Soprana, P.R. Madonie, 37 $51^{\prime} 55^{\prime \prime}$ N, 1405'17" E, 1300 m, 13 Oct. 2002, Ilex, Quercus, Fagus, Ginster, leg. L. Behne (SDEI); 1 q, Etna Süd Zafferano, Casa Tomacchio, 20 Jun. 2001, 1200 m, leg. D. Siede (SDEI); 1 ô, 2 ex., Umgeb. Taormina,

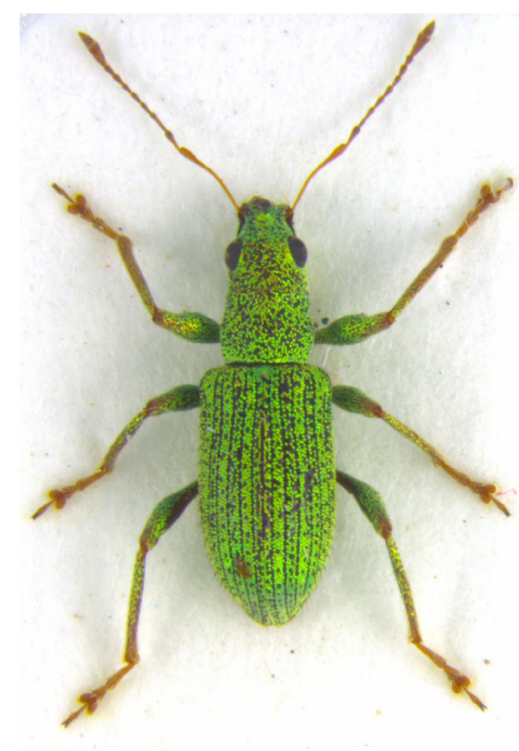

A

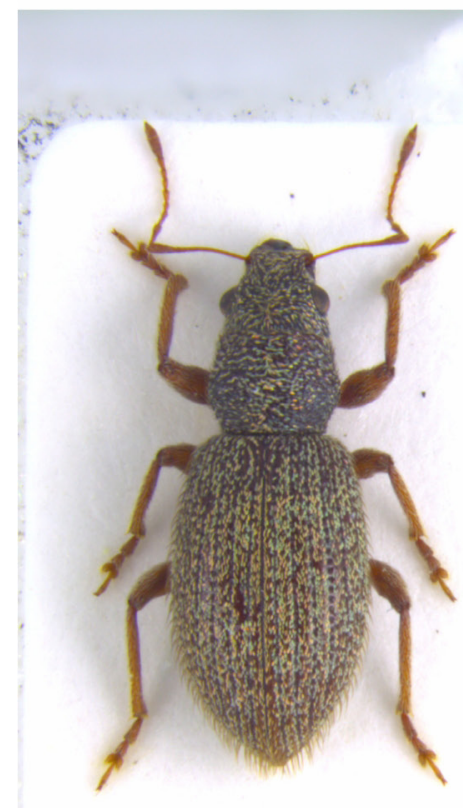

D
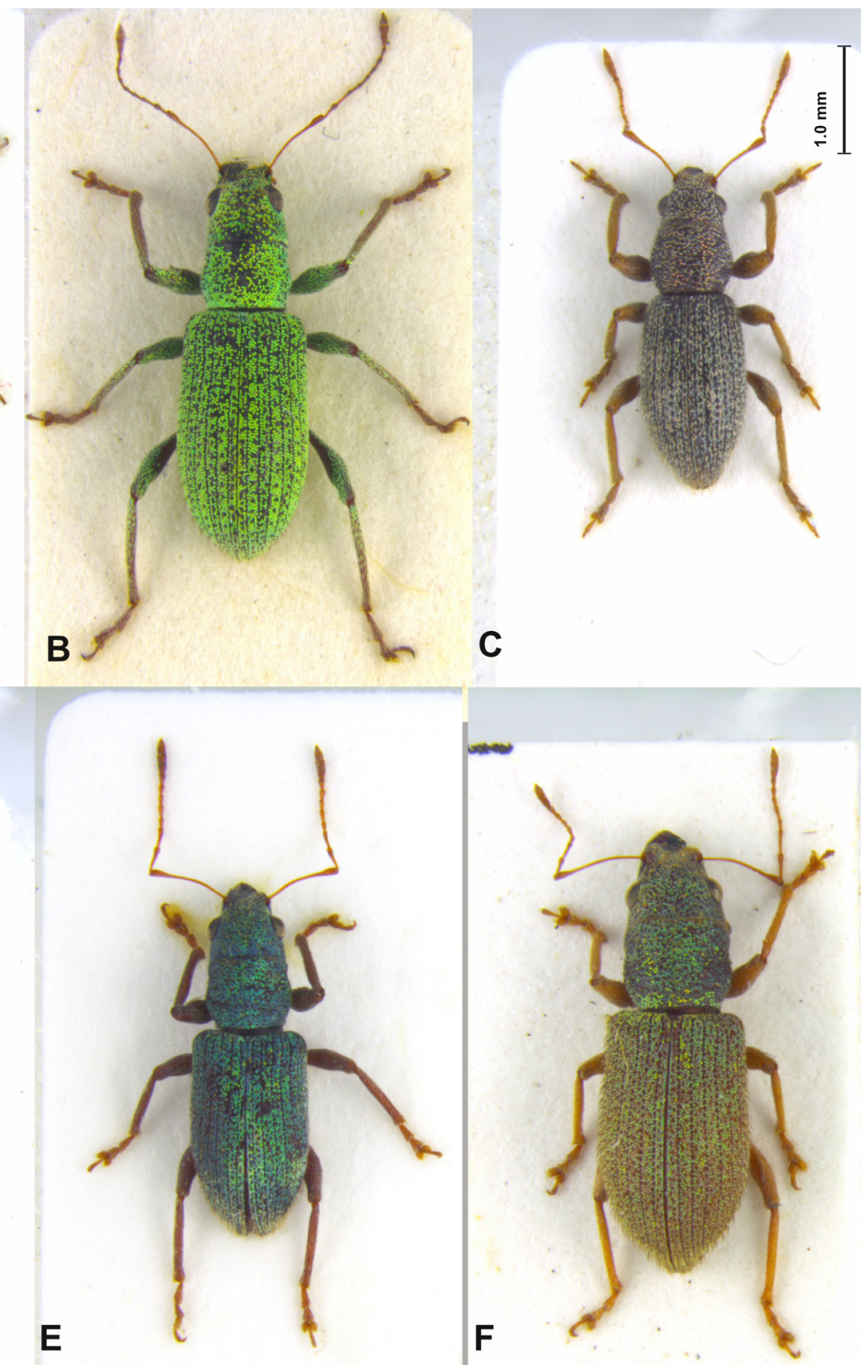

Fig. 1. A-B. Habitus of Polydrusus (Denticonocetus) kahri Kirsch, 1865. A. Male from Ancona, Italy. B. Female from Sicily, Italy. - C-D. Polydrusus (Conocetus) festae (F. Solari, 1925) from Samos Island, Greece. C. Male. D. Female. - E-F. Polydrusus stierlini (Schilsky, 1910). E. Male from Chalkidike, Greece. F. Female from Peloponnese, Greece. 
Sic., 21 Apr.-8 May [19]42, leg. Gg. \& Ht. Frey (NMB); 2 ex., Monte Baldo, coll. J.B. Jörger (NMB); 6 ex., Ancona, leg. Flach (SDEI); 1 đ, 1 ex., Abruzzen (NMB); 3 ex., Roveredo [Rovereto], Halbherr, coll. L.v. Heyden (SDEI); 1 ㅇ, Apennin, Umgb. Florenz, 14-25 Aug. 1987 (cJM).

\section{Comments on new synonymies}

Based on all specimens examined and their identical internal sacs (see Fig. 9A-C), P. siculus and $P$. vodozi are hereby regarded as junior synonyms of $P$. kahri.

Magnano (1964) resurrected P. siculus from synonymy, mainly based on the differences given by Desbrochers des Loges (1872) in the original description. However, upon examination of specimens from various localities for the present study, these characters do not support a species status, therefore Polydrusus siculus $=$ Polydrusus kahri.

In the case of $P$. vodozi, the synonymy with $P$. kahri proposed by Hoffmann (1950) and Schott (2014) is formally approved here (Polydrusus vodozi $=$ Polydrusus kahri). The type specimen in the collection of Desbrochers was examined by L. Schott and confirmed as conspecific with P. kahri; furthermore, a male specimen from Corsica was examined (see below).

\section{Remarks}

Polydrusus kahri is a widespread species reported from France (Alpes Maritimes), including Corsica (Vizzavona: Hoffmann 1950; Schott 2014), Austria to Dalmatia, Bosnia-Herzegovina, Macedonia, Montenegro (Yunakov 2013; no specimens presently examined and not included in Fig. 10), Greece, and all of Italy including Sicily, but apparently excluding Sardinia (mentioned by Hoffmann 1950, but not included in Colonnelli 2003, nor in Abbazzi \& Maggini 2009). Records by Yunakov (2013) from Cyprus are highly dubious and may refer to misidentifications. Polydrusus kahri shows large variability in size and density of vestiture.

Polydrusus (Conocetus) crinipes sp. nov. urn:1sid:zoobank.org:act:897EA3D6-350A-4C5A-8E6C-02CF14DCF3E8

Figs 3A-B, 7E, 9G, 11

\section{Etymology}

The name 'crinipes' derives from the characteristic hairs 'crinis' on the legs '-pes', shared only with P. rhodiacus within the subgenus.

\section{Type material}

\section{Holotype}

GREECE: §̊, Korfu, Airportlagune, 28 Jul. 1989, leg. W. Suppantschitsch (SDEI) // [red label:] Holotype Polydrusus (Conocetus) crinipes sp. nov. des. C. Germann 2016 (SDEI).

Type locality

GREECE: Corfu Island.

\section{Paratypes}

BULGARIA: 1 ơ, Pirin, Sugarevo, 700-900 m, 25 Jun. 1983, leg. R. Borovec (cRB); 1 \&, Sandanski,

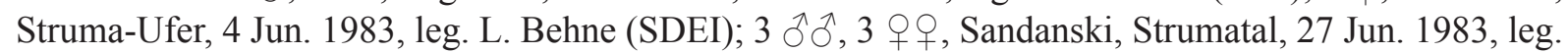
L. Behne (SDEI, cRB); 2 $\widehat{\jmath}, 6$ 우, Sandanski, Strumaufer, 4 Jun. 1983, leg. L. Behne (SDEI); 1 ð, Pirin, Kasina, 1300-1400 m, 28 Jun. 1983, leg. R. Borovec (cRB); 1 đ’, 1 q, Sandanski, Jun. 1970, leg. D. Marek (cJF). 
GREECE: 13 ex., same data as holotype (SDEI, NMBE, cCG); 2 ex., Korfu, Ropa Tal, 25 Jul. 1989, leg. W. Suppantschitsch (SDEI); 6 ex., Corfu, leg. Paganetti (SDEI); 1 ex., Corfu, leg. Paganetti (cJF); 2 ex. Corfu, leg. Paganetti (BMNH); 1 ô, Corfu, leg. Paganetti (NMBE); 1 ex., Corfu, leg. Paganetti (cRB); 1 ex., Corfu, Roda, 19 May-2 Jun. 2012, leg. Bahr \& Winkelmann (cHW); 1 ð, Corfu, Mt Pandokratoras, Lafki, 420 m, 21 May 2012, leg. Bahr and Winkelmann (cHW); 2 ex., Corfu, Agios Georgios, 6-14 Jun. 2002, leg. K. Schön (cRB); 1 ex., Corfu, Agios Georgios, 19-27 Jun. 2003, leg. L. Korission and K. Schön (cRB); 2 ex., Corfu, Roda, 16-23 Jun. 1995, leg. Czetö Zsolt (cRB); 1 ex., Corfu, Agios

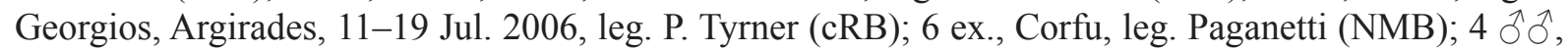
1 +, Macedonia, Piera, Paralia, 1-15 Jun. 2013, leg. F. Bahr, J. Messutat and H. Winkelmann (cJM); 1 ex., Macedonia, Piera, Paralia, 15 Jun. 2013, leg. F. Bahr, J. Messutat and H. Winkelmann (cHW);

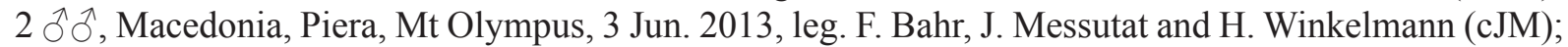

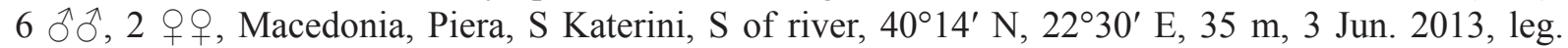
F. Bahr, J. Messutat and H. Winkelmann (cJM); 3 ex., C-Maced. (FO3) Piera, Katerini S, 3 Jun. 2013, F. Bahr, J. Messutat and H.Winkelmann (cJM); 1 ô, 1 q, Macedonia, Piera, Paralia, beach, 6-9 Jun. 2013, leg. F. Bahr, J. Messutat and H. Winkelmann (cJM); 1 §, Nestos-Delta, Chrysoupolis, 29 Jun. 1982, leg. Kessler (SDEI); 1 q, Nestos-Delta, Chrysoupolis, 30 Jun. 1982, leg. Kessler (SDEI); 1 q, Macedonia, Nestos-Delta, Chrysoupolis, 26 Jun. 1982, leg. Kessler (SDEI); 1 ○, Polikastro, 8 km N of Axioupoli, 28 Jun. 1997, leg. C. Bayer (cHW); 2 $\lesssim, 2$ 우우, Polikastro NW, 8 km N of Axioupoli, 28 Jun.

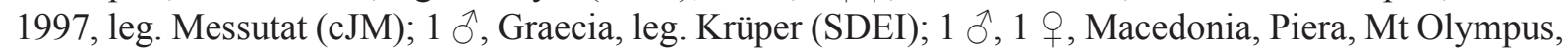
600-1050 m, 3 Jun. 2013, leg. F. Bahr, J. Messutat and H. Winkelmann (cHW); 2 ex., Olympos, Plaka, 12-14 Jul. 1985, leg. W. Suppantschitsch (SDEI); 1 ex., Trikala, n. Kalambaka (Fluss), leg. W. Ziegler, 11 Jun. 2012 (cHW); 1 ex., Nestos-Delta, 10 km E of Nea Karia, 4053'37" N, 2450'17" E, 20 m, 9 Jul. 2003, leg. Bahr, Bayer and Winkelmann (FO8); 1 ex., Prov. Thesprotia, Kestrini b. Igoumenitsa, 6 Jun.

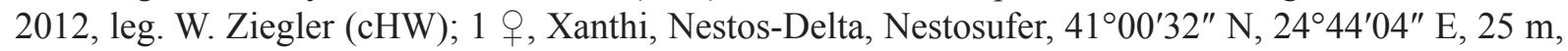
29 May 2016, leg. C. Germann (cCG); 1 Ô, 1 ๆ, Xanthi, Nestos-Delta, N Chrysoupoli, Ruderalfläche, $41^{\circ} 00^{\prime} 46^{\prime \prime} \mathrm{N}, 24^{\circ} 42^{\prime} 39^{\prime \prime}$ E, 40 m, 29 May 2016, leg. C. Germann (cCG); 1 ô, 3 q o, Xanthi, 2.5 km NE

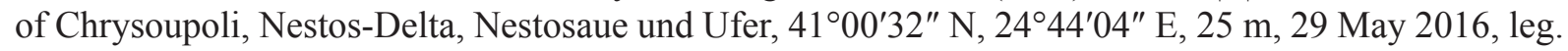
C. Braunert (cCB).

\section{Description}

Length. 3.7-4.3 mm, holotype $4.2 \mathrm{~mm}$; females $4.2-5.0 \mathrm{~mm}$.

CoLour. Body colour reddish-brown. Head, pronotum and elytra set with oval to roundish, vivid green shimmering scales and long, pointed, light brownish adjacent hairs.

HEAD. Rostrum short and wide, almost twice as wide as long. Eyes oval, irregularly bulged, strongest in first third, well protruding from outline of head. Pterygia well developed, visible from above. Pterygial span as wide as frons just behind the eyes. Rostral dorsum and frons between the eyes flat, upper side of head behind eyes slightly bulged. Rostral dorsum irregularly striato-punctuate with carinula in the middle. Epistome well visible, V-shaped, margin glossy. Mandibles strong, left mandible above right one, thus fore margin of epistome assymmetrically protruding leftwards. Antennal scrobes lateral, short and triangular, lower margin buckled downwards, with obtuse angle. Temples visible, $1 / 2$ the maximal eye length.

ANTENNAE. Long and slender, antennal funiculus longer than scape, reddish-brown, scape bowed, clubbed towards apex, reaching backwards into first third of pronotum. Antennal funiculus with 7 antennomeres: $1^{\text {st }}$ longer than $2^{\text {nd }}$ and $3^{\text {rd, }} ; 5^{\text {th }}$ to $7^{\text {th }}$ of same length; $4^{\text {th }}$ slightly longer than $3^{\text {rd }}$ and $5^{\text {th }}$ to $7^{\text {th }}$; club with 3 segments, elongate-oval.

Pronotum. Transverse (L/W: 0.7-0.8), sides slightly rounded, widest just behind the middle, constricted just before fore margin and simply rounded towards hind margin. Hairs and oval to lanceolate greenish 
scales directing towards an imaginary line in the middle of the disc. Scales at disc of pronotum narrower oval.

ELYTRA. L/W: males 2.0, females 1.8-1.9. Elongate, widest at shoulders and almost parallel (males) or widest behind the middle (females), round-pointed towards apex, shoulders present, with hind wings. Striae with shorter, adjacent white hairs. Interspaces with long, light brownish, adjacent, pointed hairs standing irregularly. Vestiture consisting of regular-standing broad oval to roundish scales with vivid green shimmer. Scales narrower along suture.

LEGS. Strong, reddish-brown coloured to yellowish, all femora edentate, set with whitish hairs with a pearl-like lustre. Tarsi with first tarsomere twice as long as second, third bilobed, about 1.5 times as wide as the second, fourth one minute, claw segment as long as second and third together, claws fused at base.

MALE GENITALIA. Penis with apophyses shorter than median lobe, this attenuated towards broad triangular apex with blunt tip, in lateral view flattened (Fig. 7E). Internal sac wrench-like, with leaf-like flattened base, two triangular sclerites at apex and a thin, tube-like, bowed sclerite in-between in lateral view (Fig. 9G).

Female Genitalia. Spiculum ventrale with very long and slender apodeme, plate hardly sclerotized. Spermatheca C-shaped with short nodulus and ramus; nodulus twice as long as ramus.

\title{
Remarks, variability and differentiation
}

The new species corresponds to specimens misidentified by authors as Polydrusus bardus. Examination of the holotype of $P$. bardus revealed that the description by Gyllenhal (in Schoenherr et al. 1834) referred instead to a species commonly known as P. gracilicornis Kiesenwetter, 1864 (or its junior synonyms, see the overview above for details), and therefore has priority over that name.

The reddish-brown to yellowish legs, with hairs but deprived of scales, are characteristic for both P. crinipes sp. nov. and P. rhodiacus. Polydrusus crinipes sp. nov. further differs from $P$. rhodiacus by the protruding convex eyes (Fig. 3A-B) (vs elongate drop-shaped in P. rhodiacus; Fig. 2E-F).

\section{Ecology}

The new species was collected from May to July, often along rivers (e.g., Nestos River), from sea level to montane altitudes, on various deciduous trees (e.g., Corylus avellana, Populus, Ulmus, Ziziphus).

\author{
Polydrusus (Conocetus) transjordanus sp. nov. \\ urn:1sid:zoobank.org:act:CFF6EFCB-C8C1-489E-BBF0-794D18269536
}

Figs 2C-D, 6E-H, 9O, 11

\section{Etymology}

The name of the new species, transjordanus, is derived from its distribution across the Jordan River.

\section{Material examined}

\section{Holotype}

ISREAL: 1 đ̆, Har Meron, 5 Jun. 1974, leg. D. Furth // [red label:] Holotype Polydrusus (Conocetus) transjordanus sp. nov. des. C. Germann 2016 (SMNHTAU).

Type locality

ISRAEL: Mount Meron. 


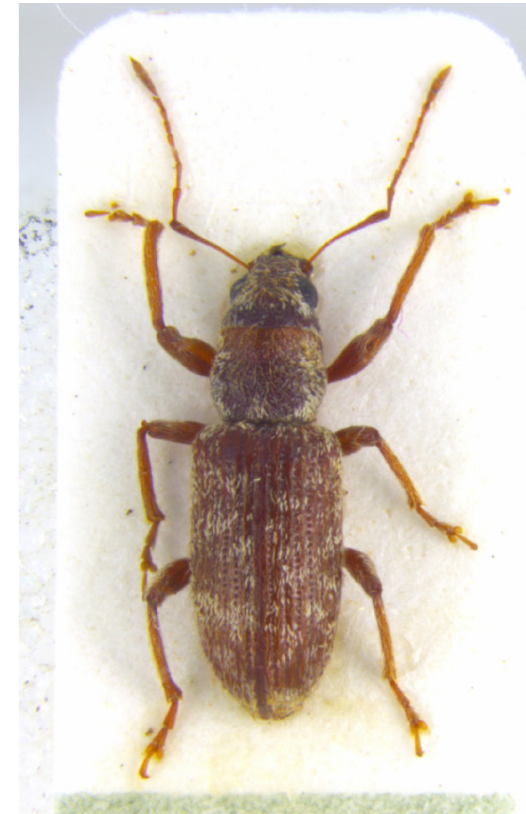

A

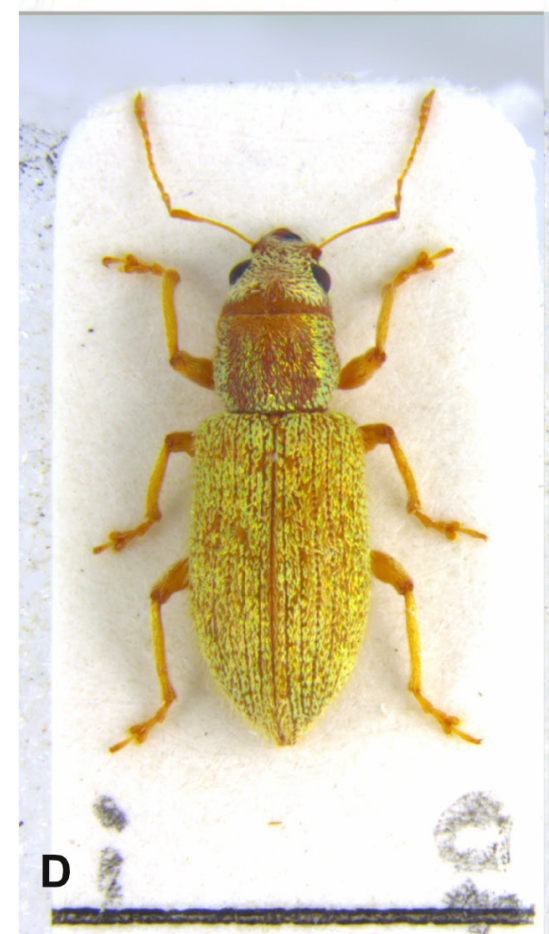

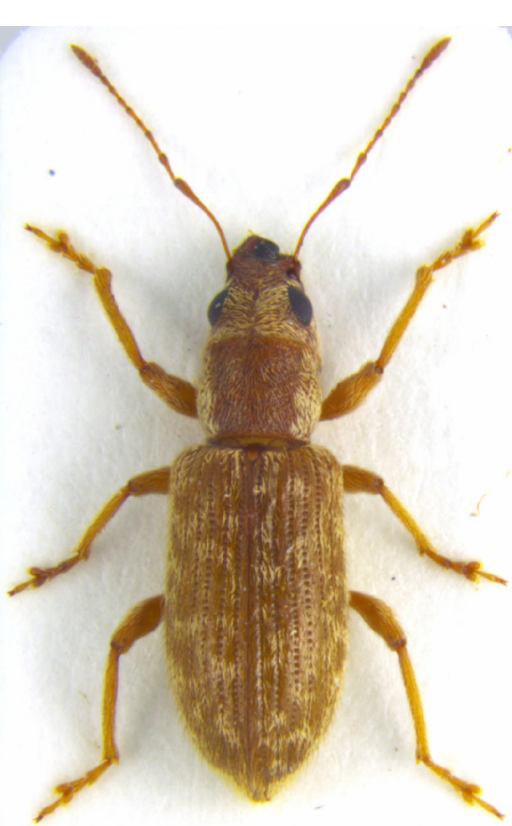

B

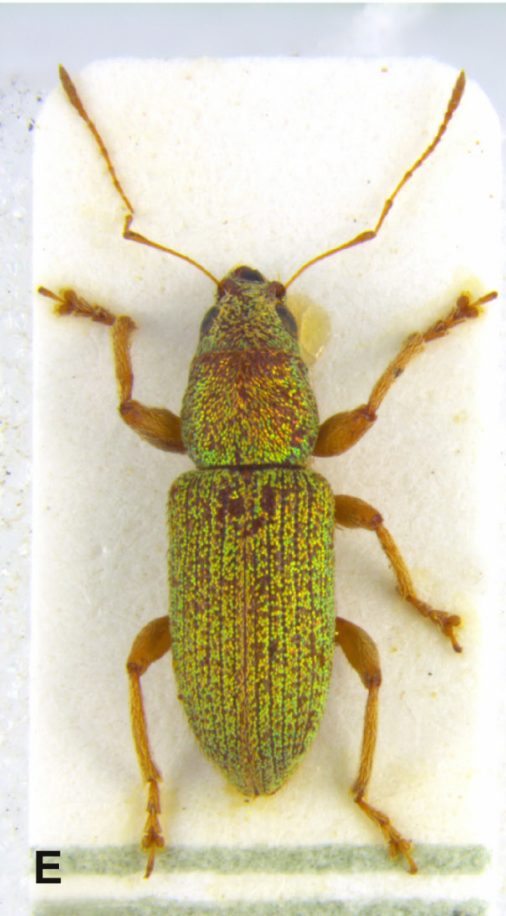

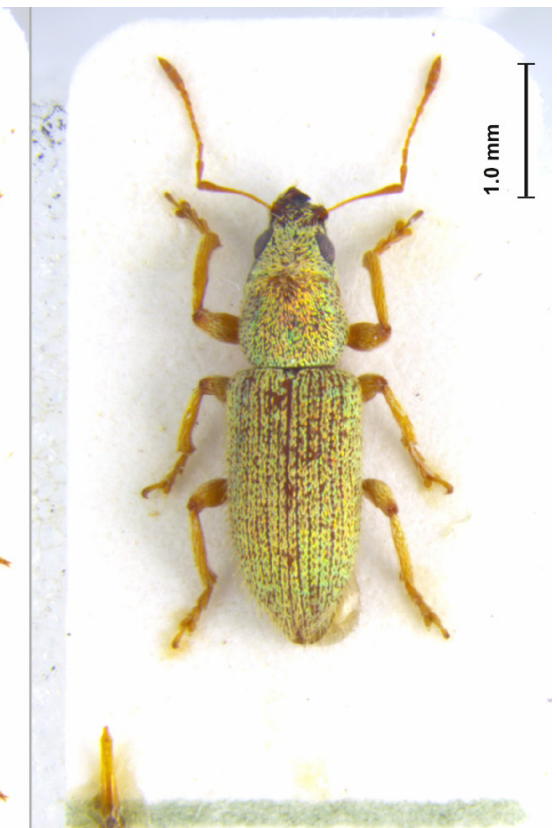

C

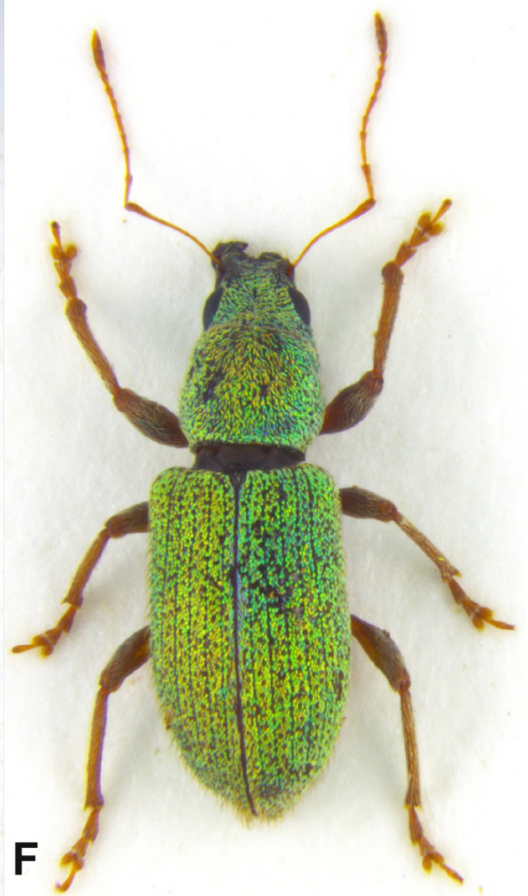

Fig. 2. A-B. Habitus of Polydrusus (Conocetus) grandiceps (Desbrochers des Loges, 1875). A. Lectotype male from Lebanon. B. Female from Syria. - C-D. Polydrusus transjordanus sp. nov., paratypes from Jordan. C. Male. D. Female. - E-F. Polydrusus rhodiacus (Schilsky, 1912). E. Male from Nicosia, Cyprus. F. Female lectotype from Cyprus. 


\section{Paratypes}

In addition to the colletion data cited below, the paratype specimens all carry the following red label: Paratype Polydrusus (Conocetus) transjordanus sp. nov. des. C. Germann 2016.

ISRAEL: 6 ex., same data as holotype (SMNHTAU, cCG); 3 ex., Meron, 1 Jun. 1978, leg. D. Furth (SMNHTAU); 1 §̂, Kaifa (= Haifa), leg. Reitter (MFN); 1 ex., Qiryat Tiv'on, 25 Apr. 1954, on Quercus ithaburensis, coll. M. Sternlicht, coll. G.A.K. Marshall (BMNH); 1 §̃, Kibuz Dahlia, 15 May 1975, leg.

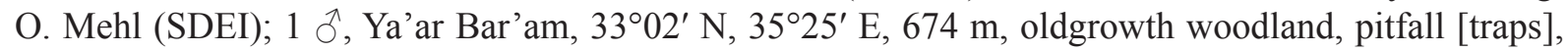
19 Aug. 2005, leg. A. Timm and T. Assmann (SMNHTAU); 1 đે, Mt Meron, Ziv'on, 22 Nov. 2007, leg. Levanony (SMNHTAU); $1 \hat{\jmath}$, Nahal, Oren, riverbed, 11 May 2003, leg. A. Freidberg (SMNHTAU); 2 ex., Hurfeish, recent woodland, $32^{\circ} 60^{\prime} \mathrm{N}, 35^{\circ} 22^{\prime}$ E, pitfall [traps], 6 May 2005, leg. A. Timm and T. Assmann (SMNHTAU); 1 ex., Hurfeish, recent woodland, $32^{\circ} 60^{\prime} \mathrm{N}, 35^{\circ} 22^{\prime} \mathrm{E}, 706 \mathrm{~m}$, pitfall [traps], 25 Jun. 2005, leg. A. Timm and T. Assmann (SMNHTAU); 4 ex., Hurfeish, recent woodland, $32^{\circ} 60^{\prime} \mathrm{N}$, $35^{\circ} 22^{\prime}$ E, 706 m, pitfall [traps], 4 Jul. 2005, leg. A. Timm and T. Assmann (SMNHTAU); 3 ex., Ya'ar Bar'am, $33^{\circ} 02^{\prime} \mathrm{N}, 35^{\circ} 25^{\prime} \mathrm{E}, 674 \mathrm{~m}$, oldgrowth woodland, pitfall [traps], $22 \mathrm{Jul}$. 2005, leg. A. Timm and

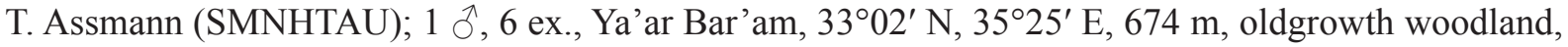
pitfall [traps], 7 Jul. 2005, leg. A. Timm and T. Assmann (SMNHTAU); 6 ex., Ziv'on, 33 $02^{\prime} \mathrm{N}, 35^{\circ} 25^{\prime} \mathrm{E}$, recent woodland, $773 \mathrm{~m}$, pitfall [traps], 4 Jun. 2005, leg. A. Timm \& T. Assmann (SMNHTAU); 4 ex., Ya' ar Bar'am, $33^{\circ} 02^{\prime} \mathrm{N}, 35^{\circ} 25^{\prime} \mathrm{E}, 674 \mathrm{~m}$, oldgrowth woodland, pitfall [traps], 4 Aug. 2005, leg. A. Timm

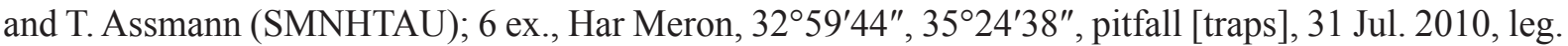
L. Friedman and C. Drees (SMNHTAU); 1 ex., Hurfeish, $32^{\circ} 60^{\prime}$ N, $35^{\circ} 22^{\prime}$ E, recent woodland, pitfall [traps], 21 Jan. 2006, leg. A. Timm and T. Assmann (SMNHTAU); 1 ex., Ya'ar Bar'am, 330' ${ }^{\prime} \mathrm{N}, 35^{\circ} 25^{\prime}$ E, 674 m, oldgrowth woodland, pitfall [traps], 25 Jun. 2005, leg. A. Timm and T. Assmann (SMNHTAU); 2 ex., Mt Meron, Zi'on, 22 Nov. 2007, leg. T. Levanony (SMNHTAU); 1 ex., Nahal Keziv, 3302'43"

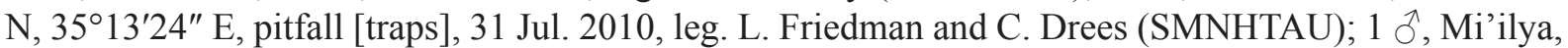
on Quercus, 23 Jun. 1987, leg. Y. Zvik (SMNHTAU); 2 ex., Har Meron, 32 $60^{\prime}$ N, 35²5' E, 1140 m, 26 Jun. 2014, leg. A. Freidberg (SMNHTAU); 1 ex., Har Addir, 980 m, on Quercus boissieri, Q. calliprinos, 20 May 2013, leg. L. Friedman (SMNHTAU); 2 ex., Har Meron, 800 m, Field School, 18 Aug. 2000, leg. L. Friedman (SMNHTAU); 1 ex., Har Meron, Jan. 2010, leg. V. Kravchenko (SMNHTAU); 1 ex., Galilee Nahal Keziv, 20 May 1999, leg. Chikatunov (SMNHTAU); 1 ex., Har Meron, 3259'44" N, $35^{\circ} 24^{\prime} 38^{\prime \prime}$ E, pitfall [traps], 31 Jul. 2010, leg. L. Friedman and C. Drees (SMNHTAU); 1 ex., Ma'alot, on Quercus calliprinos, 16 Aug. 1990, leg. J. Halperin (SMNHTAU); 6 ex., Har Meron, 1100 m, 6 Sep. 2007, leg. L. Friedman (SMNHTAU); 1 ex., Har Meron, 2006, leg. H. Tsegai (SMNHTAU); 1 ex., Har Meron, 1100 m, 23 Sep. 1997, leg. L. Friedman (SMNHTAU); 3 ex., Har Meron, 1100 m, 26 May 1999, leg. L. Friedman (SMNHTAU); 1 ex., Har Meron, pitfall, 28 May 2006, leg. N. Angel, (SMNHTAU); 2 ex., Har Meron, 800 m, 28 May 2003, leg. L. Friedman (SMNHTAU); 1 ex., Har Meron, 800 m, 22 May 1998, leg. L. Friedman (SMNHTAU); 1 ex., Har Meron, 19 May 1998, leg. L. Friedman (SMNHTAU); 2 ex., Har Meron, 1100 m, 26 May 1999, leg. L. Friedman (SMNHTAU); 1 ex., Har Meron, 800 m, 26 May 1999, leg. O. Manheim (SMNHTAU); 1 ex., Mt Meron, 5 Oct. 1976, leg. A. Freidberg (SMNHTAU); 1 ex., Mt Meron, 1200 m, 3 Jul. 1980, leg. D. Furth (SMNHTAU); 4 ex., Meron, 1 Jun. 1978, leg. D. Furth (SMNHTAU); 3 ex., N-Keziv, 8 May 1979, leg. D. Furth (SMNHTAU);

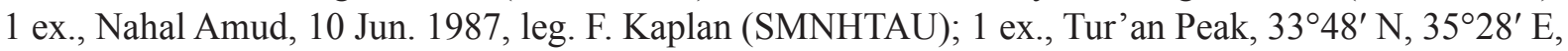
555 m, 18 Apr. 2010, leg. A. Freidberg (SMNHTAU); 2 ex., Mi'ilya, on Quercus, 23 Jun. 1987, leg. Y. Zvik (SMNHTAU); 2 ex., Har Addir, 980 m, , on Q. boissieri, Q. calliprinos, 20 May 2013, leg. L. Friedman (SMNHTAU); 6 ex., Mazzuva, Nahal Bezet, 60 m, 20 May 2013, leg. L. Friedman, on Q. calliprinos (SMNHTAU); 1 ex., Hurfeish, $32^{\circ} 60^{\prime} \mathrm{N}, 35^{\circ} 22^{\prime} \mathrm{E}$, recent woodland, pitfall, 12 Oct. 2005 , leg. A. Timm and T. Assmann (SMNHTAU); 4 ex., Har Meron, 32 $2^{\circ} 60^{\prime} \mathrm{N}, 35^{\circ} 25^{\prime} \mathrm{E}, 1140 \mathrm{~m}, 26$ Jun. 2014, leg. A. Freidberg (SMNHTAU); 1 ex., Meron, 10 Sep. 1973, leg. D. Furth; 4 ex., Nahal Oren, $32^{\circ} 42^{\prime} 53.9^{\prime \prime}$ N, 3458'35.8" E, 2 May 2002, leg. A. Freidberg (SMNHTAU); 1 ex., Nahal Oren, 17 May

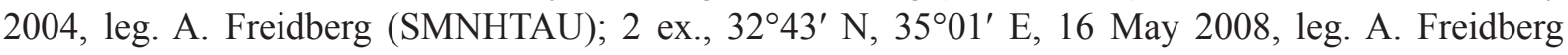


(SMNHTAU); 5 ex., Nahal Oren, 14 May 2002, leg. A. Freidberg (SMNHTAU); 2 ex., Nahal Oren, 22 May 2001, leg. L. Friedman (SMNHTAU); 2 ex., Nahal Kelah, 22 May 2001, leg. L. Friedman (SMNHTAU); 1 ex., Nahal Oren, 4 May 2004, leg. I. Zonstein (SMNHTAU); 5 ex., Nahal Oren, riverbed, 2 May 2002 leg. L. Friedman (SMNHTAU); 1 ex., Nahal Oren, riverbed, 14 May 2003 leg. L. Friedman (SMNHTAU); 4 ex., Nahal Oren, 31 May 2002, leg. A. Freidberg (SMNHTAU); 1 ex., Nahal Oren, 2 Jul. 1996, leg. L. Friedman (SMNHTAU); 1 ex., Nahal Oren, riverbed, 11 May 2003, leg. A. Freidberg (SMNHTAU); 2 ex., Nahal Oren, 1 May 2001, leg. L. Friedman (SMNHTAU); 1 ex., Nahal Oren, 16 May 1999, leg. I. Lopatin (SMNHTAU); 6 ex., Nahal Oren, 32 $43.5^{\prime}$ N, $35^{\circ} 00.5^{\prime}$ E,

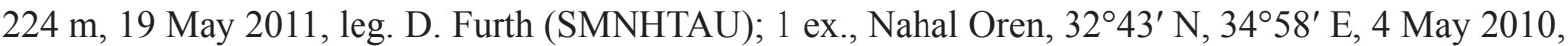
leg. W. Kuslitzky (SMNHTAU); 12 ex., Nahal Oren, Karmiyya Ridge, 2 May 2002, leg. L. Friedman

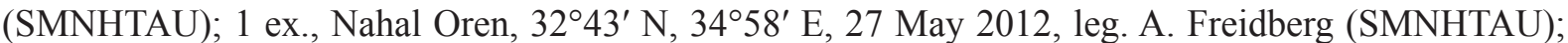

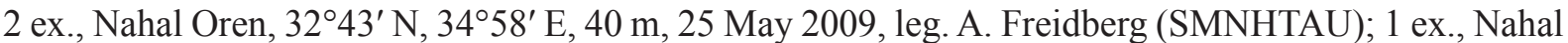

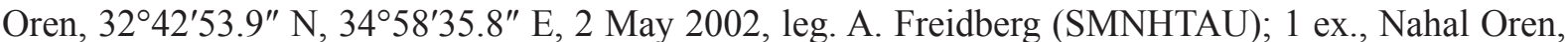
30 Apr. 1974, leg. D. Furth (SMNHTAU); 1 ex., Nahal Keziv, 28 May 1999, leg. M. Finkel (SMNHTAU); 1 ex., Nahal Oren, riverbed, 11 May 2003, leg. A. Freidberg (SMNHTAU); 17 ex., Nahal Oren, 1 May 2001, leg. L. Friedman (SMNHTAU); 1 ex., Nahal Oren, 21 Apr. 2007, leg. A. Freidberg (SMNHTAU); 1 ex., Nahal Oren, 24 Apr. 2004, leg. A. Freidberg (SMNHTAU); 2 ex., Mt Tabor, 14 May 1979, leg.

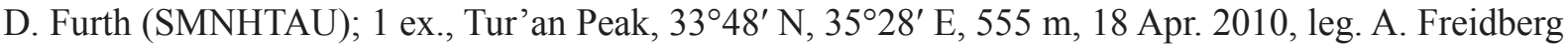
(SMNHTAU); 1 ex., Hay-Bar Karmel, 450 m, 21 May 2010, leg. A. Freidberg (SMNHTAU); 1 ex., Zomet Ha' Amaqim (Jalame), 26-30 May 1993, leg. A. Freidberg (SMNHTAU); 2 ex., Yoqne'am, 28 Apr. 1975, on Quercus ithaburensis, leg. J. Halperin (SMNHTAU); 1 ex., Yerushalayim, 15 Apr. 1958, leg. J. Halperin (SMNHTAU); 10 ex, Yizhar, Salman Farsi Nat. Res., 800 m, on Quercus calliprinos, 14 May 2014, leg. L. Friedman (SMNHTAU); 7 ex., Mazzuva, Nahal Bezet, 60 m, on Q. calliprinos, 20 May 2013, leg. L. Friedman (SMNHTAU); 1 ex., Nahal Keziv, 20 Feb. 1999, leg. M. Finkel (SMNHTAU); 5 ex., Nahal Keziv, 7 May 1999, leg. M. Finkel (SMNHTAU); 4 ex., Nahal Keziv, 20 May 1999, leg. M. Finkel (SMNHTAU); 3 ex., Nahal Keziv, 21 May 1999, leg. M. Finkel (SMNHTAU); 5 ex., Nahal Keziv, 28 May 1999, leg. M. Finkel (SMNHTAU); 1 ex., Nahal Keziv, 3 Sep. 1999, leg. M. Finkel (SMNHTAU); 1 ex., Nahal Keziv, 4 Jun. 1999, leg. M. Finkel (SMNHTAU); 1 ex., Nahal Keziv, 10 Jun. 1999, leg. M. Finkel (SMNHTAU); 3 ex., Nahal Keziv, 17 Jun. 2000, leg. M. Finkel (SMNHTAU); 3 ex., Nahal Keziv, 26 Jun. 1999, leg. M. Finkel (SMNHTAU); 1 ex., Nahal Keziv, 10 Jul. 1999, leg. M. Finkel (SMNHTAU); 1 ex., Nahal Keziv, 13 May 2000, leg. M. Finkel (SMNHTAU); 1 ex., Nahal Keziv, 21 May 1999, leg. M. Finkel (SMNHTAU); 2 ex., Nahal Keziv, 8 May 1979, leg. D. Furth (SMNHTAU); 1 ex., Mt Meron, 1200 m, 3 Jul. 1980, leg. D. Furth (SMNHTAU); 1 ex., Mt Meron, 8 Aug. 1972, leg. D. Furth (SMNHTAU); 5 ex., Meron, 5 Jun. 1974, leg. D. Furth (SMNHTAU); 2 ex., Meron, 1 Jun. 1978, leg. D. Furth (SMNHTAU); 1 ex., Meron, 10 Sep. 1973, leg. D. Furth (SMNHTAU); 12 ex., Newe Ativ, 4 Jun. 1974, leg. D. Furth (SMNHTAU); 1 ex., Shoresh, on Pistacia palaestina, 20 May 1984, leg. J. Halperin (SMNHTAU); 2 ex., Shoresh, 20 May 1978, leg. D. Furth (SMNHTAU); 2 ex., Mi'ilya, on Quercus, 23 Jun. 1987, leg. Zvik (SMNHTAU); 1 ex., Har Hermon, 1970-1990, leg. M. Warburg (SMNHTAU); 1 ex., Har Hermon, 1450 m, 25 Jun. 1997, leg. V. Chikatunov (SMNHTAU). 3 ex., Nahal Oren, 24 May 1995, leg. A. Freidberg (SMNHTAU); 2 ex., Nahal Oren, 30 May 1995, leg. A. Freidberg (SMNHTAU); 4 ex., Nahal Oren, 13 May 2007, leg.

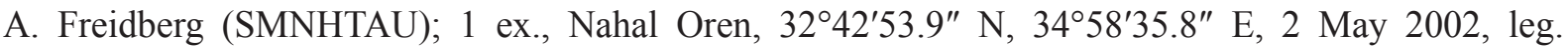
A. Freidberg (SMNHTAU); 1 ex., Nahal Oren, 9 Jun. 1978, leg. D. Furth (SMNHTAU); 1 ex., Nahal Oren, 4 May 1980, leg. D. Furth (SMNHTAU); 3 ex., Nahal Oren, 9 May 1979, leg. D. Furth (SMNHTAU); 2 ex., Nahal Oren, 9 Jun. 1978, leg. D. Furth (SMNHTAU); 1 ex., Nahal Oren, 3 Jul. 1997, leg. V. Chikatunov and T. Pavlicek (SMNHTAU); 16 ex., Nahal Oren, Mt Carmel, 27 May 1997, leg. V. Chikatunov and T. Pavlicek (SMNHTAU, BMNH); 4 ex., Nahal Oren, Mt Carmel, 13 May 1997, leg. V. Chikatunov and T. Pavlicek (SMNHTAU); 13 ex., Nahal Oren, Mt Carmel, 28 May 1996, leg. V. Chikatunov and T. Pavlicek (SMNHTAU, NMBE, NMSO); 1 ex., Nahal Oren, Mt Carmel, 11 Jun. 1996, leg. V. Chikatunov and T. Pavlicek (SMNHTAU); 6 ex., Nahal Oren, Mt Carmel, 9 Jun. 1997, leg. 
V. Chikatunov and T. Pavlicek (SMNHTAU); 1 §, Beit Oren, 12 May 1991, leg. Y. Zvik (SMNHTAU); 8 ex., N.[ahal] Oren, 10 Jul. 1997, leg. V. Chikatunov and T. Pavlicek (SMNHTAU); 1 ex., Nahal Oren, Mt Carmel, Jun. 1997, leg. V. Chikatunov and T. Pavlicek (SMNHTAU); 8 ex., Nahal Oren, Mt Carmel, 1 Jun. 1998, leg. A. Freidberg (SMNHTAU); 32 ex., Nahal Oren, Jun. 1996, leg. V. Chikatunov and T. Pavlicek (SMNHTAU, BMNH, NMBE); 2 ex., Nahal Oren, 15 Jun. 1998, leg. V. Chikatunov and T. Pavlicek (SMNHTAU); 8 ex., Nahal Oren, 18 May 1998, leg. V. Chikatunov and T. Pavlicek (SMNHTAU); 7 ex., N.[ahal] Oren, 2 Jun. 1998, leg. V. Chikatunov and T. Pavlicek (SMNHTAU); 2 ex., N.[ahal] Oren, 19 Jun. 1998, leg. V. Chikatunov and T. Pavlicek (SMNHTAU); 1 ex., Nahal Oren, Carmel [Mt], light [trap], 15 May 1997, leg. V. Chikatunov and T. Pavlicek (SMNHTAU); 1 ex., Nahal Oren, light trap, 5 Jul. 1999, leg. V. Chikatunov and T. Pavlicek (SMNHTAU); 1 ex., Nahal Oren, 26 Jun. 1997, leg. V. Chikatunov and T. Pavlicek (SMNHTAU); 1 ex., Nahal Oren, light trap, 4 Jun. 1999, leg. V. Chikatunov and T. Pavlicek (SMNHTAU); 1 ex., Qiryat Tiv'on, on Quercus ithaburensis, 18 May 1954, leg. M. Sternlicht (SMNHTAU).

JORDAN: 1 đ, Jordanie, 5 km NE of Ajlun, 32.36754 N, 35.77937 E, 1005 m, 10 Jun. 2011, leg.

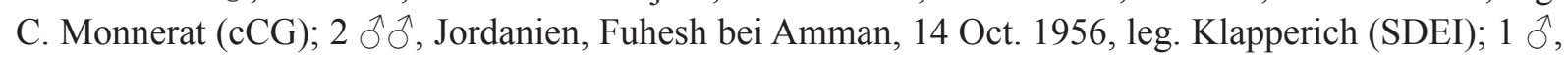
Jordanien-O, Dehbeen b. Jerash, 19 Jun. 1959, leg. J. Klapperich (SDEI); 1 \&, Ost-Jordanien, Wald bei Jerash, 800 m, 24 May 1956, leg. J. Klapperich (SDEI); 1 ð’, 1 ㅇ, Jordania, NE, Um Qais, 3240’ N, 3541' E, 9 m, 14 Apr. 2008, leg. C. Monnerat (cCG, NMBE); 1 q, Ost-Jordanien, Schaubak [Shobak], 17 May 1968, leg. Klapperich (SDEI).

\section{Additional material}

The following specimens were not included in the type series due to being partly immature or inflexible/ hardly remountable.

ISREAL: 2 ex., Yoqne'am, 28 Apr. 1975, on Quercus ithaburensis, leg. J. Halperin (SMNHTAU); 11 ex., Yizhar, Salman Farsi Nat. Res., 818 m, on Q. calliprinos, 11 Jun. 2013, leg. L. Friedman (SMNHTAU); 1 ex., Panyas, $33^{\circ} 15^{\prime}$ N, $35^{\circ} 42^{\prime}$ E, 5 Jun. 2002, leg. L. Friedman (SMNHTAU); 2 ex., Har Meron, 1100 m, 26 May 1999, leg. L. Friedman (SMNHTAU); 2 ex., Mazzuva, Nahal Bezet, 60 m, on Q. calliprinos, 20 May 2013, leg. L. Friedman (SMNHTAU); 1 ex., Har Meron, 32 ${ }^{\circ} 0^{\prime}$ N, 35²4' E, 900 m, Field School, 26 May 2009, leg. A. Freidberg (SMNHTAU); 1 ex., Bet Qeshet, Quercus ithaburensis forest, 7 Apr. 2013, leg. L. Friedman (SMNHTAU); 2 ex., Berekhat Ya'ar, 14 May 2003, leg. L. Friedman (SMNHTAU); 6 ex., Qiryat Tiv'on, on Quercus ithaburensis, 1 May 1954, leg. M. Sternlicht (SMNHTAU); 1 ex., Qiryat Tiv'on, on Quercus ithaburensis, 13 May 1954, leg. M. Sternlicht (SMNHTAU); 3 ex., Qiryat Tiv'on, on Quercus ithaburensis, 19 May 1954, leg. M. Sternlicht (SMNHTAU); 4 ex., Adullam, 15 May 2006, leg. E. Groner and V. Chikatunov (SMNHTAU); 1 ex., Har Meron, 1100 m, 26 May 1999, leg. L. Friedman (SMNHTAU); 1 ex., Har Meron, 800 m, 22 May 1999, leg. L. Friedman (SMNHTAU); 1 ex., Har Meron, pitfall [traps], 31 Jul. 2010, leg. L. Friedman and C. Drees (SMNHTAU); 2 ex. [1 imm.], Har Meron, pitfall [traps], 24 May 2006, leg. N. Angel (SMNHTAU); 12 ex., N. Sansan, 17 May [20]02, leg. Y Mandelik and V. Chikatunov (SMNHTAU); 1 ex. [imm.], Har Hermon, 1970-1990, leg. M. Warburg (SMNHTAU); 2 ex., Galilee, Nahal Keziv, 20 May 1999, leg. Chikatunov (SMNHTAU); 5 ex., Matta', 31 May 1974, leg. D. Furth (SMNHTAU).

\section{Description}

LeNGTH. Males: 3.2-4.5 mm, holotype: $3.4 \mathrm{~mm}$; females: $3.8-5.4 \mathrm{~mm}$.

BoDy. Colour reddish-brown. Vestiture of head, pronotum and elytra set with elongate-oval scales with a greenish pearl-like lustre and long, pointed, whitish hairs, adjacent on head and pronotum, semi-raised ( $25^{\circ}$ angle) on elytra. 


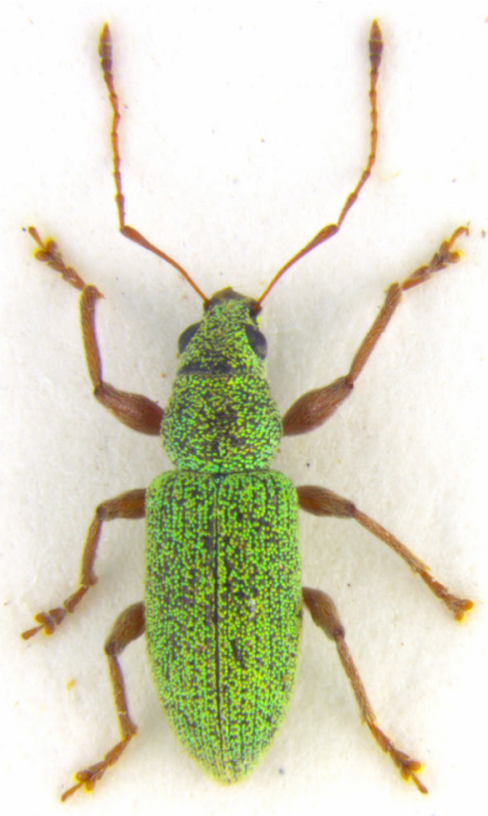

A

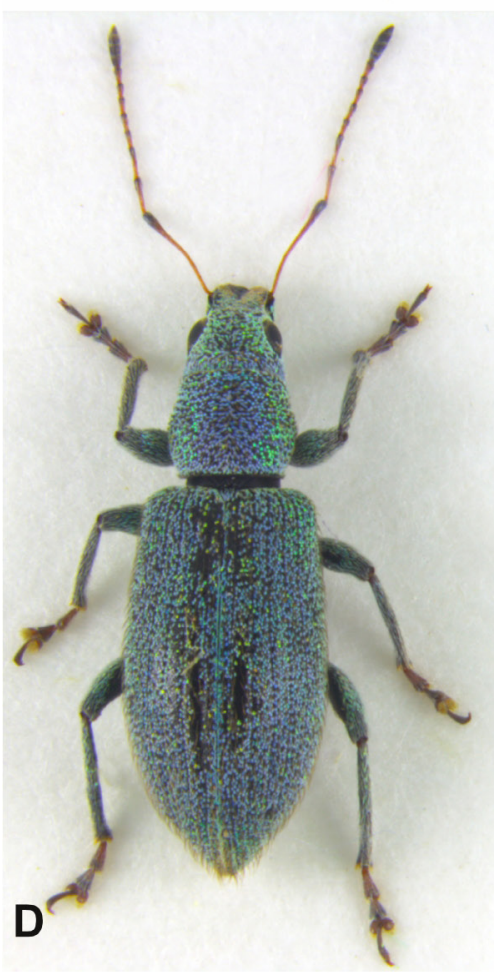

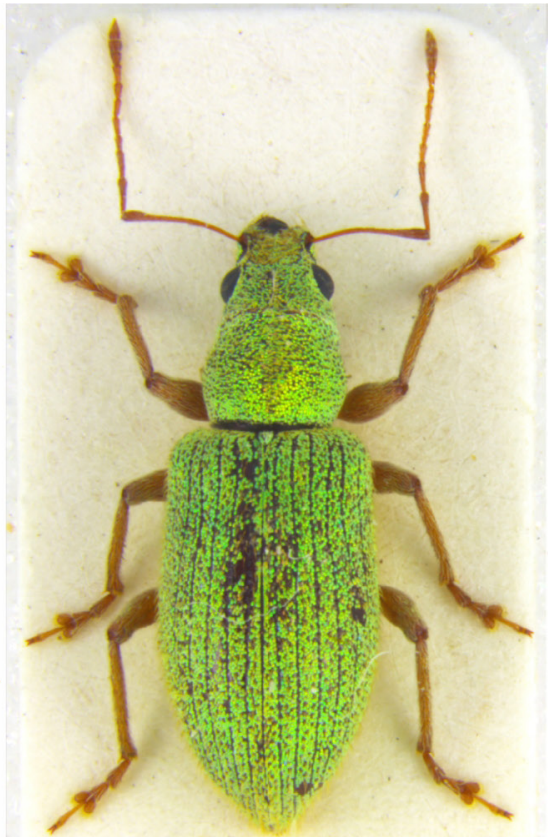

B

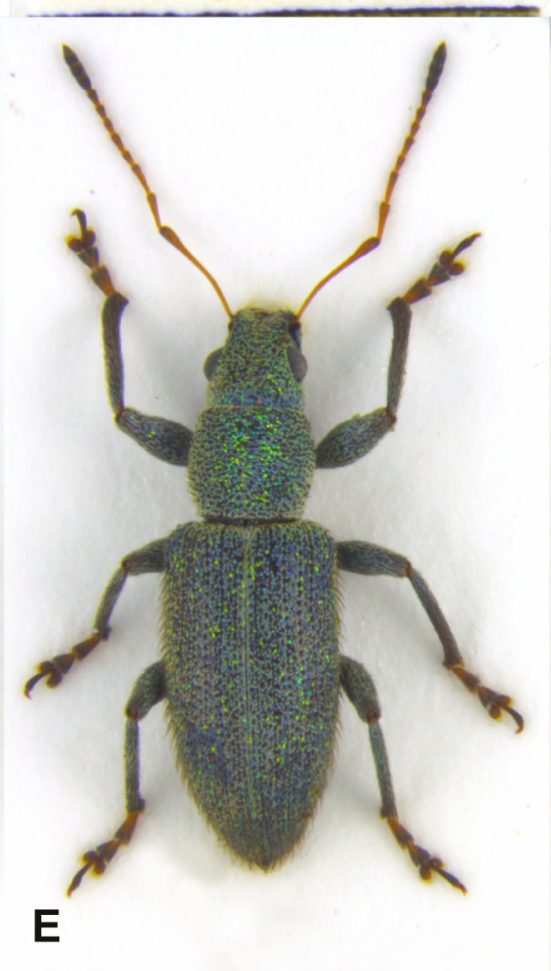

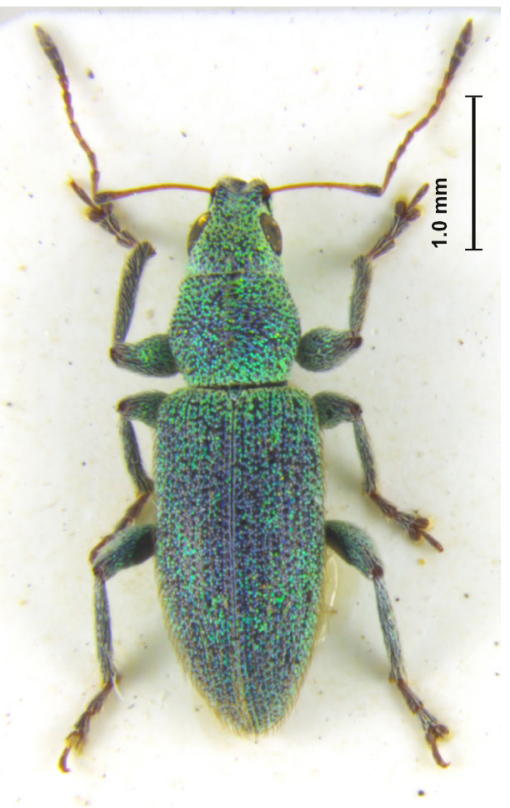

C

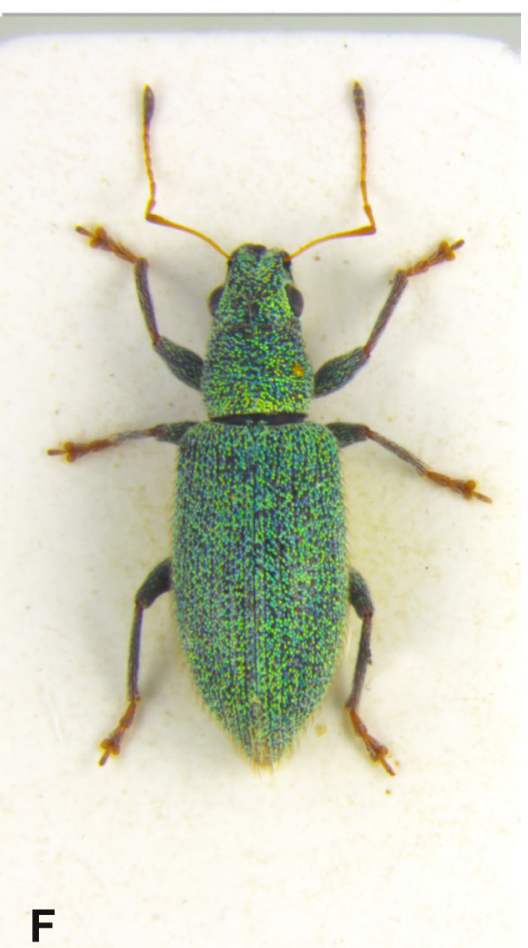

Fig. 3. A-B. Habitus of Polydrusus (Conocetus) crinipes sp. nov. A. Holotype, male from Corfu, Greece. B. Female from Bulgaria. - C-D. Polydrusus angustus (Lucas, 1854). C. Male from Rhodes, Greece. D. Female from Kalymnos, Greece. - E-F. Polydrusus calabricus (Faust, 1890). E. Male from Peloponnese, Greece. F. Female from Cephalonia, Greece. 
HeAd. Rostrum short and almost twice as wide as long. Eyes oval, regularly and moderately bulged, protruding from outline of head. Pterygia well developed, visible from above. Pterygial span as wide as frons just behind the eyes. Rostral dorsum and frons between the eyes flat, upper side of head behind eyes slightly bulged. Rostral dorsum irregularly striato-punctuate with carinula in the middle. Epistome well visible, V-shaped, margin glossy. Mandibles strong, left mandible above right one, thus fore margin of epistome asymmetrically protruding to the left. Antennal scrobes lateral, short and triangular, lower margin buckled downwards, obtuse angled. Temples visible, $2 / 3$ of the maximal eye length.

ANTENNAE. Long and slender, yellowish, scape bowed, clubbed towards apex, just reaching backwards (laterally) to fore margin of pronotum. Antennal funiculus with 7 antennomeres: $1^{\text {st }}$ longer than $2^{\text {nd }} ; 3^{\text {rd }}$ to $7^{\text {th }}$ of same length but slightly broadening; club with 3 visible segments, elongate-oval.

PRonotum. Simply rounded towards hind margin. Hairs and oval to lanceolate greenish scales directing towards imaginary point in middle of fore margin. Scales at margins of pronotum mostly standing more densely.

ELYTRA. L/W: 1.7-1.8. Elongate, widest at shoulders and parallel (males) or widest behind middle (females), round-pointed towards apex, shoulders present, with hind wings. Striae with short, adjacent white hairs as long as puncture diameter. Interspaces with long, whitish, semi-raised and pointed hairs standing regularly in one row. Vestiture appearing spotty and consisting of oval to elongate-oval scales with greenish pearl-like lustre. Scales narrower and smaller along suture.

LEGS. Strong, reddish-brown to yellowish coloured, all femora edentate, set with whitish hairs and oval scales with a greenish pearl-like lustre. Tarsi with first tarsomere twice as long as second, third bilobed, about 1.5 times as wide as second, fourth one minute, claw segment as long as second and third together, claws fused at base.

Male genitalia. Penis with apophyses shorter than median lobe, this attenuated up to $2 / 3$ of its length, from there broadening towards triangular apex with prolonged blunt tip protruding tongue-like, flattened in lateral view (Fig. 6E-H). Internal sac wrench-like, with leaf-like flattened base, two S-shaped sclerites at apex and a strong, b-shaped sclerite in-between in lateral view (Fig. 9O).

Female Genitalia. Spiculum ventrale with very long and slender apodeme, plate hardly sclerotized. Spermatheca C-shaped with short nodulus and slightly longer, strongly bowed ramus.

\section{Variability and differentiation}

The density of the vestiture varies and the colour of the scales varies from greenish to whitish, but always with a pearl-like lustre. The size also varies considerably: males are generally smaller than females, but the eyes of males are bigger, in relation to head size, than those of females. Polydrusus (Conocetus) transjordanus sp. nov. is morphologically most similar to P. grandiceps, differing in the smaller eyes (in relation to head size; Fig. 5A-B), mostly more greenish and more oval scales, and the more gracile penis (see key; Fig. 6E-H). Polydrusus bardus is at first sight morphologically similar to $P$. transjordanus sp. nov. and may reach the distribution area of the latter at the north-western border (ancient records from "Syria"; see under bardus). However, the vestiture of $P$. bardus consists of denser and more regular standing, roundish and more vivid-green scales on the elytra and pronotum (loosely standing, oval and pale greenish ones in P. transjordanus sp. nov.), and shorter, more adjacent hairs on the head, pronotum and elytra (about twice as long and more steeply raised in P. transjordanus sp. nov.). 


\section{Ecology}

The new species was found almost year-round (from January to November) and collected mainly in woodland on both evergreen and summer-green oaks (e.g., Quercus ilex, Q. boissieri, Q. calliprinos, Q. ithaburensis) and on Pistacia palaestina, from lowlands up to montane altitudes.

\section{Polydrusus (Conocetus) angustus (Lucas, 1854)}

Figs 3C-D, 7F, 9H, 11

\section{Material examined}

\section{Syntypes}

Two syntypes of Polydrusus virens (Kiesenwetter, 1864):

GREECE: 1 + , Athens // Kiesenwetter (ZSM); 1 đ, virens Ksw. Grèce Ksw. // Celadonius (Phyll.) Brull. $\widehat{o}$ Polydrosus [sic!] (ZSM). [Both with additional red labels:] Syntype Polydrusus (Conocetus) virens (Kiesenwetter, 1864) labelled C. Germann 2016.

Two syntypes of Polydrusus (Conocetus) femoratus (Stierlin, 1888):

GREECE: 1 §, Griechenland [handwritten by L. Dieckmann] // Syntypus [red label] // coll. Stierlin // Polydrusus femoratus Stierl. det. Dieckmann 1989 (SDEI); 1 đ, Griechenld // P. femoratus Stl. [both Stierlin's handwriting] // Syntypus // Polydrusus femoratus Stierl. det. Dieckmann 1989 (SDEI).

\section{Type locality}

GREECE: Mount Ida, Crete.

\section{Other material examined}

GREECE: 1 \%, Naxos, coll. Stierlin (SDEI); 4 ex., Graecia coll. Stierlin (SDEI); 1 \%, Thessalia, Volo, leg. Stussiner (SDEI); 3 ex., Thessal[ia] Pelion, leg. Stussiner (SDEI); 2 ex., Aegina v. Oertzen (SDEI); $1 \hat{\jmath}$, Naxos, leg. Schatzmayr (SDEI); 2 ex., Kreta, leg. Paganetti (SDEI); 7 ex., Parnass, leg. Paganetti (SDEI); 1 ex., Attica, leg. Reitter (SDEI); $1 \curvearrowright$, Athen, coll. V. Heyden (SDEI); 1 + , Attica, leg. Reitter,

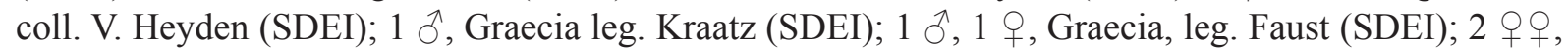
Parnass, leg. Paganetti, coll. Piesbergen (SDEI); 2 ex., Asiat. Türkei, Rhodes, coll. Stöcklein (NMB); 2 ex., Volo, Thessalien, coll. P. Meyer (NMB); 2 $\hat{\jmath}$, Parnass, Graecia (NMB); 2 ex., Cycla. [Cyclades], coll. Kraatz (SDEI); 1 ex., femoratus Strl. Graecia Zeiller (SDEI); 1 ex., Taygetos, coll. Apfelbeck (NMB); 1 ex., Graecia, coll. Apfelbeck (NMB); 2 ex., Parnass, leg. Paganetti, coll. J. Breit (NMB); 3 ex., Parnass, leg. Paganetti 1903, coll. Stöcklein (NMB); 2 ex., Kreta, leg. Paganetti, coll. Stöcklein (NMB); 1 ex., Parnass, Paganetti, coll. G.A.K. Marshall (BMNH); 1 §, Parnass, Paganetti, coll. J.B. Jörger (NMB); 2 ex., Parnass, Paganetti, coll. J. Breit (NMB); 1 ex., Kreta, Paganetti, coll. G.A.K. Marshall (BMNH); 1 ex., Paleohoka, 17 May 1999, Crète, C. Besnard rec. (cJP); 4 ex., Saloniki, coll. A. Rätzer (NMBE); 1 ex., Kreta Ins., Dikti Mts, Lesithi plateau, 800 m, 5 Jun. 2001, leg. J. Vořŕšsek (BMNH); 2 ex., Rhodos, 11-30 May 1974, v. Budberg leg. (BMNH); 1 o, Peloponesos, S of Tripolis, Karies, 24 Jun. 2004, J. Voříšek leg. (BMNH); 1 \&, Peloponesos or., Astros, 5-20 m, 25 May 2004, J. Voříšek leg. (BMNH); 1 ex., Rhodos, 9 May [19]70, leg. Dr. Engelman, coll O. Voříšek (BMNH); 2 ô, Attika, Varkiza, 6 May [19]93, leg. Colonnelli (cRB); 21 ex., Rhodes, Lachania, env. 50 m, 8 May 1995, leg. R. Borovec (cCG, cRB); 1 ex., Rhodes (FO 13), Afandou beach, 5 May [20]14, leg. J. Messutat (cJM); 18 ex., Rhodes, Kalithea env. (FO 1), 24 Apr.-7 May 2014, leg. J. Messutat (cJM); 4 ex., Rhodes (FO 2), Kolymbia, beach, 26 Apr. [20]14, leg. J. Messutat (cJM); 8 ex., Rhodes (FO 7), Apollona, N, 400 m, 29 Apr. [20]14, leg. J. Messutat (cJM); 1 ex., Rhodes (FO 3), Archipoli NE, 150 m, 26 Apr. [20]14, leg. J. Messutat (cJM); 1 ex., Rhodes (FO 6), Laerma N, 260 m, 28 Apr. [20]14, leg. J. Messutat 
(cJM); 7 ex., Rhodes (FO 9), Soroni-Fanes, 30 m, 1 May [20]14, leg. J. Messutat (cJM); 1 ex., Rhodes (FO 12), Genadi, beach, 3 May [20]14, leg. J. Messutat (cJM); Rhodes (FO 11), Emponas, 380 m, 3 May [20]14, leg. J. Messutat (cJM); 3 ex., Rhodes (FO 13), Afandou, beach, 5 May [20]14, leg. J. Messutat (cJM); 1 đ, 1 ㅇ, Agios Nikolao, Kreta, 24 Apr. 1971, W. Wittmer (NMB); 1 ex., Crete, Chania, Potamida,

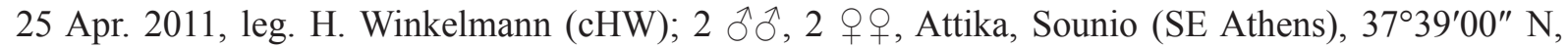
2401'40" E, 50 m, 17 May 2011, leg. Bahr, Bayer, Brunner and Winkelmann (cHW); 1 ex., Peloponnes, Kaliani, Prov. Korinthia, 5 May 2010, leg. W. Ziegler (cHW); 1 ex., Prov. Drama, Prosotsani, 20 Jul. 2009, leg. W. Ziegler (cHW); 1 ex., Prov. Korinthia, Stimfalia, 13 May 2007, leg. W. Ziegler (cHW); 1 $\widehat{\jmath}$, Rhodos, Kallithea, 5-19 May 2005, leg. S. Scharf (cHW); 2 우, Kreta, Knossos Umgb., Jun. [19]92, leg. H.-J. Grunwald (cHW); 3 ex., Platistomo, 31 May 2013, $38^{\circ} 58^{\prime}$ N, $22^{\circ} 07^{\prime}$ E, leg. Eifler (cHW); 1 J, Peloponnese, Argolis, Mt Didymo, Didyma, doline, 6 Apr. 2010, leg. H. Winkelmann (cHW); 1 q, Peloponnese, Argolis, Palea Epidavros, 1-10 Apr. 2010, leg. H. Winkelmann (cHW); 1 q, Peloponnese, Corinthia, Korfos env., 10 Apr. 2010, leg. H. Winkelmann (cHW); 2 ô, Crete, Chania, Potamida, 25 Apr. 2011, leg. H. Winkelmann (cHW); 1 , Peloponnes, Galatas, 7-28 Jun. [19]76, Köstlin leg. (SDEI); 1 9, Samos, May [19]87, leg. Zimmerman (SDEI); 18 ex., Rhodes, Kalithea env. (FO1), 24 Apr.-7 May 2014, leg. Messutat (cCG, cJM, NMBE); 1 , Rhodes (FO 12), Genadi, beach,

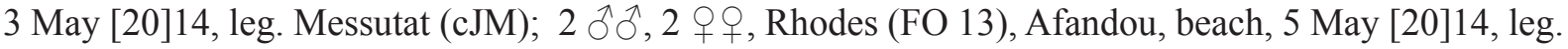
Messutat (cJM); 1 \%, Rhodes (FO 11), Emponas, 380 m, 3 May [20]14, leg. Messutat (cJM); 8 ex., Rhodes (FO 7), Apollona N, 400 m, 29 Apr .[20]14, leg. Messutat (cJM); 4 ex., Rhodes (FO 2), Kolymbia, beach, 26 Apr. [20]14, leg. Messutat (cJM); 1 ex., Rhodes (FO 3), Archipoli NE, 150 m, 26 Apr. [20]14, leg. Messutat (cJM); 1 \%, Rhodes (FO6), Laerma N, 260 m, 28 Apr. [20]14, leg. Messutat (cJM); 5 ex., Rhodes (FO 9), Soroni-Fanes, 30 m, 1 May [20]14, leg. Messutat (NMBE); 6 ex., N Peloponnesus, Kato

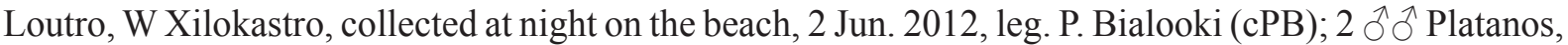
NE Parnon Mts, 18 May 2012, leg. P. Bialooki (cPB); 1 \%, Gerakini, 4 Jun. 1992, leg. V. Svilha (cJS); 1 J, Pelop. [onnese], Mt Taygetos, Asafigion, 24 Jun. [1974], Horak \& Svihla leg. (cJS); 4 우, Nomos, Lakonias, $5 \mathrm{~km} \mathrm{~S}$ of Monemvasia, 13-25 May [19]89, leg. Rieger (cCG, cJM); 1 ○, Makedonia-S, $6 \mathrm{~km}$

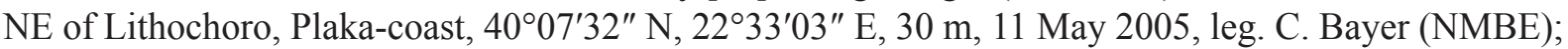
1 ๙, 1 q, Rhodes, coll. Stöcklein (NMB); 2 우, Thessalien, Volo, coll. P. Meyer (NMB); 2 $\delta$, Parnass (NMB); 1 q, Taygetos, coll. Apfelbeck (NMB); 3 ex., Parnass, Paganetti [19]03, coll. Stöcklein (NMB); 1 , 1 q, Kreta, Paganetti, coll. Stöcklein (NMB); 1 ô, 1 , Kreta, Agios Nikolao, 24 Apr. 1971, leg. Wittmer (NMB); 12 ex., E Peloponnesus, pass W Astros, 18 May 2012, leg. P. Bialooki (cPB); 1 , Attica, Nea Makri N, 1-14 Jun. 2015, leg. Messutat (cJM); 1 ㅇ, Euboia, Mt Ochi, $2.5 \mathrm{~km}$ NE of Myli, 965 m, 4 Jun. 2015, leg. Messutat (cJM); 4 ex., Rhodes, Kolymbia, beach, 3 Apr. 2015, leg. Messutat (cJM); 2 ex., [Peloponnese] Nomos Lakonias, Strand, $5 \mathrm{~km} \mathrm{~N}$ of Monemvasia, 15-25 May [19]89, leg. Rieger (cJM); 15 ex., Rhodes, 5 May 1996, Paradissi Mts, 100 m, Paradisi env., R. Borovec leg. (cRB, BMNH); 2 §ึో, 2 우우, 5 ex., Rhodes, 10 May 1996, Filerimos Mts, Lalyssos, 267 m, R. Borovec leg. (BMNH); 4 ex., Attiki, Varkiza, 8 May 1993, leg. E. Colonnelli (BMNH); 1 ex., Creta,

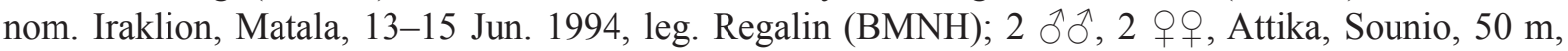
17 May 2011, leg. Bahr, Bayer, Brunner and Winkelmann (cHW); 3 ex., Crete Chania, Potamida, 25 Apr. 2011, leg. H. Winkelmann (cHW); 1 क , Peloponnes, Kaliani, Prov. Korinthia, leg. W. Ziegler, 5 May 2010 (cHW); 1 đે, Drama, Prosotsani, leg. W. Ziegler, 20 Jul. 2009 (cHW); 1 ô, Korinthia, Stimfalia, 13 May 2007, leg. W. Ziegler (cHW); 1 ô, Rhodos, Kallithea, 5-19 May 2005, leg. S. Scharf (cHW); 2 우, Kreta, Knossos Umgb., Jun. 1992, leg. H.-J. Grunwald (cHW); 3 ex., Platistomo, 31 May 2013, leg. Eifler (cHW); 1 đ, Peloponnes, Argolis, Mt Didymo, Didyma doline, 6 Apr. 2010, leg. Winkelmann (cHW); 1 o, Peloponnese, Argolis, Palea Epidavros, 1-10 Apr. 2010, leg. Winkelmann (cHW); 1 , Peloponnese, Corinthia, Korfos env., 10 Apr. 2010, leg. Winkelmann (cHW); 1 q, Peloponnes, Galatas, 7-28 Jun.[1976], leg. Köstlin (SDEI); 1 ex., Ost-Kreta, Vai, 6 Jun. 1999, leg. U. Heinig (SDEI); 1 , Süd-Kreta, Umgb. Koutsouras, 1 Jun. 1999, leg. U. Heinig (SDEI); 1 ○ૈ, Kreta, Umgb. Matala, 17-23 Apr. 2001, leg. Sieber (SDEI); 4 ex., Kreta, May [19]87, Chersonnissos, leg. R. Frieser (SDEI); 1 ex., Athen, [leg.] Schulz (SDEI); 1 Oे, Crete, Heraklion, Timbaki, 3 Apr. 2013, leg. H. Winkelmann (cHW); 
1 q. Rhodes, Afantou env., 200 m, 10 May 1996, leg. R. Borovec (cRB); 2 우, 13 ex., Rhodos, Umgb. Faliraki, E [end of] May [19]83, R. Frieser leg. (SDEI); 1 đે, Kreta, Aghios, Nikolaos 12 Apr.-3 May 1975, leg. R. Köstlin (SDEI); 1 +, Peloponnes, Astros, 14 Jun. [19]86, leg. H. Nebauer (SDEI); 1 ○, Rhodes, 7 May 1995, Koutsoutis env., pr. Emponas, 400 m, leg. Ing. K. Schön (cKS); 12 ex., Crete Isl., Rethymno, Agia Gallini, 0 m, 16 May 2009, leg. C. Germann (cCG, NMBE, NMSO); 1 ô, Crete Isl., Heraklio, N Demati (E Pirgos), N3502'28" N, E2518'01" E, 160 m, 19 May 2009, leg. C. Germann (cCG); 11 ex., Kalymnos Island, 1-6 Jun. 2012, leg. T. Kissling and M. Albrecht (cCG, NMBE, NMSO); $1 \widehat{\jmath}$, Peloponnese, Voidokilia-Bucht, Sommer [summer] 2005, leg. K. Germann (NMBE); 7 ex. Samos, 3 km NE of Pythagoreio, 20 m, 10 Jun. 2014, leg. Fouque René (cPB); 28 ex., Xanthi, Nestos-Delta, N

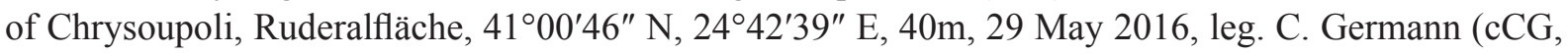
NMSO).

ITALY (first records for Italy): 13 ex., Sicilia mer. [meridionale], Cassibile, 80 m, 12-13 May 1995, beating of Amygdalus, leg. R. Borovec (cCG, cRB); 4 ex., Calabrica, leg. Baudi (SDEI); 1 ô, 1 ㅇ, Sicilia mer., Cassibille [sic!], 80 m, 12-13 May 1995, beating of Amygdalus, leg. M. Holecova (SDEI).

TURKEY: 1 ex., Asiatic Turkey, 1934, leg. Nihal Shevket (BMNH); 1 ex., Didyma, 21 May 1995, leg. Dr. A. Hamet (cRB); 1 đ’, Smyrna, coll. Apfelbeck (NMB); 8 ex., E Selendi, S Akhisar, 17 May 2010,

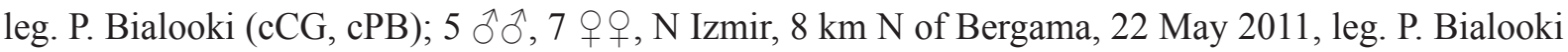
(cPB, cCG); 2 우, S Antalya, Gündogmus, 27 Sep. 1980, leg. N. Lodos (cCG, cJF); 1 + , Menavgat, 5 May 1969, leg. Wewalka (cJF); 1 , Smyrna [Izmir], coll. Apfelbeck (NMB).

\section{Variability and differentiation}

Males are more gracile than females. The colour of legs varies from dark brown and blackish to yellowish. The most similar species is Polydrusus calabricus which shares the body shape (long-oval elytra) and the shape of the penis (Fig. 7F-G). Both are easily separable by the shape of the eyes (see key and Fig. 3C-F). Only P. rhodiacus shows similar eyes (Fig. 2E-F), but differs in legs deprived of scales and the elongate shape of the penis (Fig. 7D).

\section{Ecology}

Collected from April to June (exceptionally also in September) at various altitudes from beach to montane level from different deciduous trees (Amygdalus, Pistacia lentiscus, Prunus, Quercus) and Cupressaceae.

Polydrusus (Conocetus) bardus Gyllenhal, 1834

Figs 4A-B, 7H, 9K-L, 10

\section{Material examined}

Type material

TURKEY: 1 ( (holotype of P. bardus Gyllenhal, 1834), "Chersonis" [and a second word, probably the collector "Eindstedt..." illegibly handwritten] // NHRS-JLKB 000023178 // Typus [red printed label].

GREECE: 1 \ (holotype of P. graecus (Stierlin, 1884)), Holotype Attica // coll. Stierlin // Holotypus // C. graecus Stl. // Holotypus P. graecus Stierlin det. Dieckmann 1989 (SDEI). The synonymy with P. bardus is confirmed.

\section{Type locality}

The type locality of $P$. bardus is Thracian Chersonese, today Gallipoli in Turkish Thrace. 

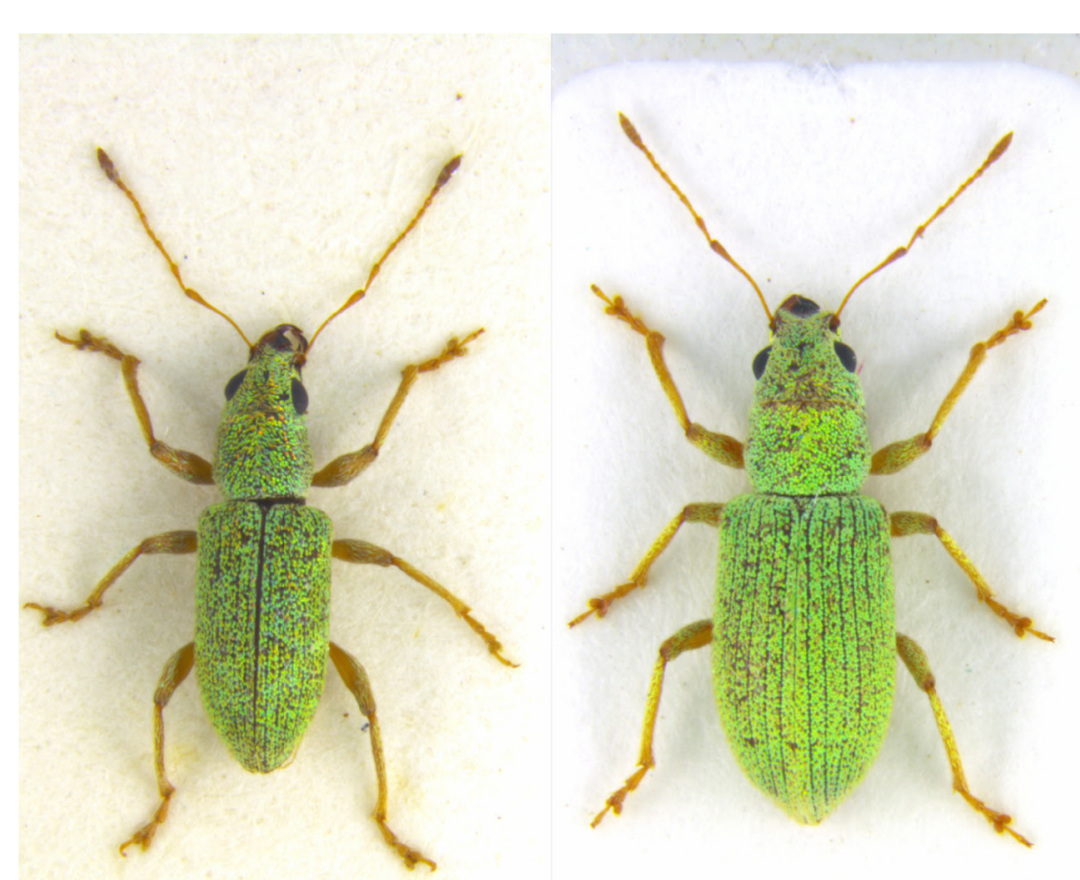

A

B
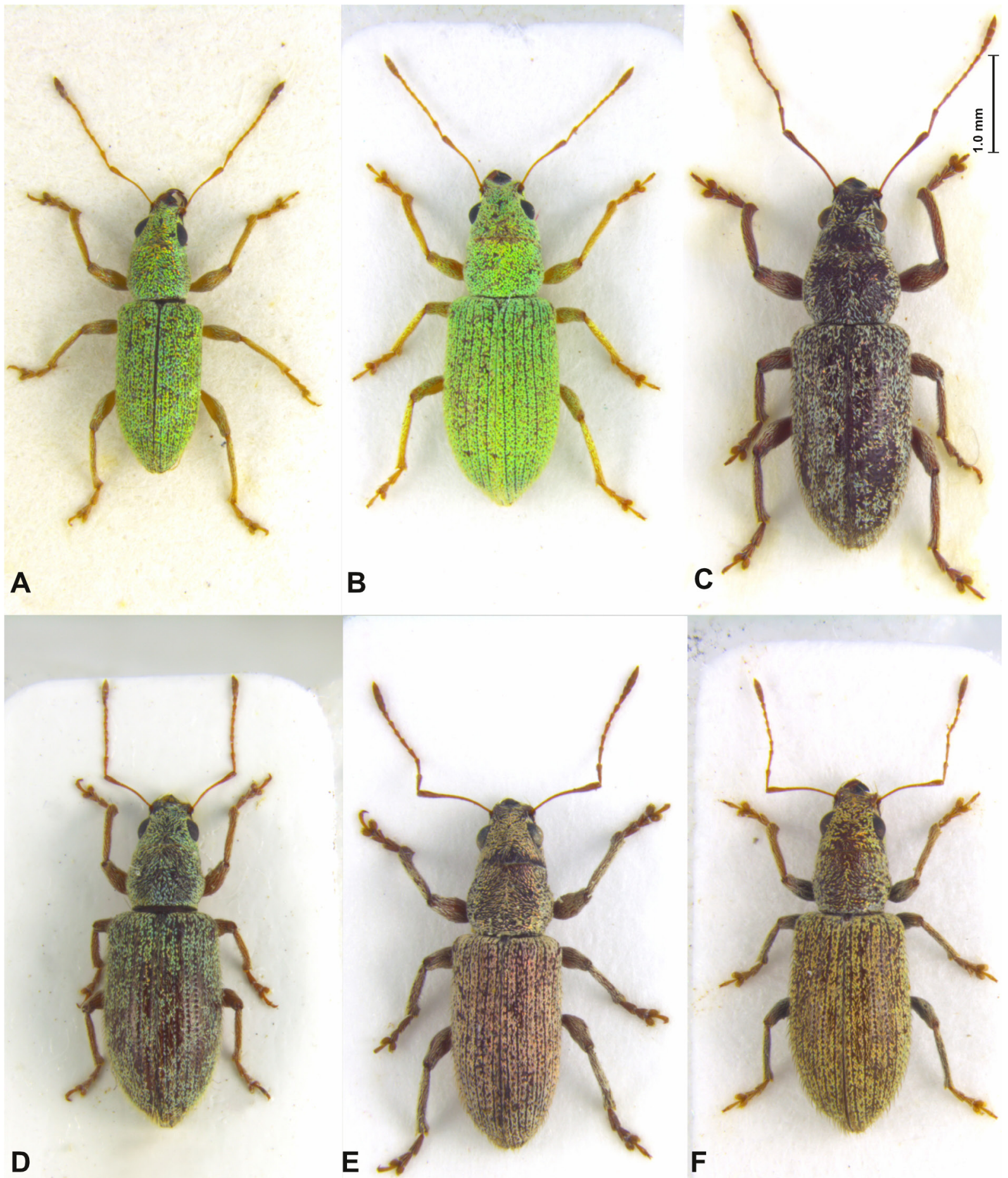

Fig. 4. A-B. Habitus of Polydrusus (Conocetus) bardus Gyllenhal, 1834. A. Male from Crete, Greece. B. Female from Rhodes, Greece. - C-D. Polydrusus marcidus Kiesenwetter, 1864. C. Male from Antalya, Turkey. D. Female from Chalkidike, Greece. - E-F. Polydrusus baudii (Faust, 1889). E. Male from Lemesos, Cyprus. F. Female from Paphos, Cyprus. 


\section{Other material examined}

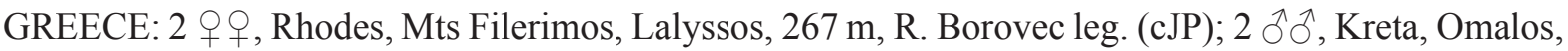
25 Jul. 2009, leg. M. Egger (NMBE, cCG); 1 \&, Crête, Therisso, 15 Apr. 1984, leg. P. Berger (cJP); $1{ }^{\dagger}$, W-Kreta, ö. Rethimno, Perama, 26 Apr. 2011, leg. W. Ziegler (cHW); 1 ex., Creta, coll. Koltze (SDEI); 1 $\widehat{ }$, Ins. Creta, Reitter (SDEI); 1 ex., Westl. Creta, Elos, v. Oertzen, coll. Kraatz (SDEI); 2 ex., Creta, Oertzen, coll. Kraatz (SDEI); 1 ðै, Creta or., Assitaes, Holtz, coll. Breit (NMB); 1 o, 1 q, Creta Isl., Rethymno, W Armeni, Kastellos, 35¹8'39" N, 2426'55" E, 430 m, 13 May 2009, leg. Germann (cCG); 1 ð W-Kreta, Georgiuopoli, May 1989, leg. Frieser (SDEI); 2 ex., [Crete] Assitaes, coll. Koltze (SDEI);

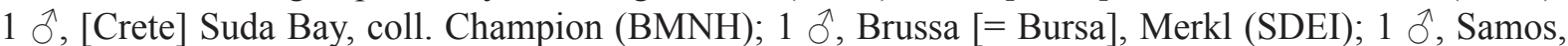
Votsalakia env., 11-20 Jun. 2007, leg. Schön (cRB); 24 ô $^{\top}$, Chalkidike, NE Megali, Panajia, 40 25'39"

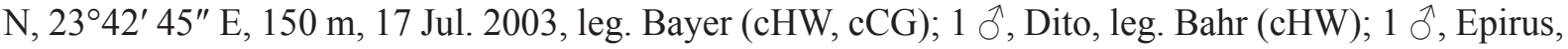
Velia Pindos, nr. Grevina, 8 Aug. 1996, leg. Barries and Cate (SDEI); 1 q, Crete, Rethimno, Armeni, 2 May 1998, leg. Thompson (BMNH); 1 q, 3 ex., Rhodes, Filerimos Mts, Lalyssos, 267 m, 10 May 1996, leg. Borovec (cRB, BMNH); 1 , , Crête, Agia Irini, 18 Jun. 1984, leg. Berger (cJP); 1 ô, Peloponnes, Messinia, Exohori, 5 Jun. 2011, leg. Ziegler (cHW); 1 đે, Pella, Agrarpidees, ssö Florina, 31 Jul. 2009, leg. Ziegler (cHW); 1 ex., Taygetos (ZSM); 1 ex., J. Rhodus [Rhodes], coll. Fremuth (cRB); 1 ô, Kreta,

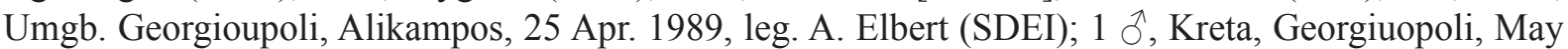

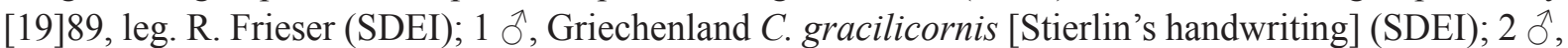

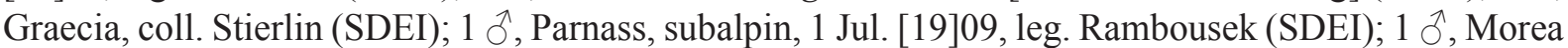
[= Peloponnese], coll. Kraatz (SDEI); 1 ô, Katerini, Ag. Dimitrios, 800 m, 19-21 Jul. 1990, leg. Schuh and Zabransky (SDEI); 2 ठ઼, Creta, coll. O. Leonhard (SDEI); 1 ex., Crete, 3 Jun. 1981, Lefka Ori Mts, Omalos, Bily leg. (cJF); 1 O$^{\lambda}$, Rhodes [leg.] Reitter (BMNH). 7 ex., Kefalonia, Agios Eleftherios, 3 km NW, 38 $11.482^{\prime} \mathrm{N}, 20^{\circ} 35.658^{\prime} \mathrm{E}, 670 \mathrm{~m}$, on Quercus ilex and Q. coccifera, $27 \mathrm{Jun} .2016$, leg. L. Friedman (SMNHTAU); 1 ex., Kefalonia, Agios Eleftherios, 5 km E, 3809.972' N, 20³9.947' E, 600 m, 27 Jun. 2016, leg. L. Friedman (SMNHTAU); 9 ex., Kefalonia, Charakti $38^{\circ} 10.310^{\prime}$ N 2041.359' E, 530 m, on Quercus ilex and Q. coccifera, 28 Jun. 2016, leg. L. Friedman (SMNHTAU); 5 ex., Kefalonia,

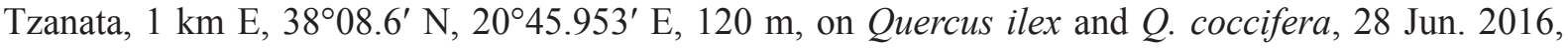
leg. L. Friedman (SMNHTAU); 34 ex., Kefalonia, Tzanata, Mycenean Tholos Tomb, $38^{\circ} 08.509^{\prime}$ N, $20^{\circ} 45.646^{\prime}$ E, $70 \mathrm{~m}$, on Quercus ilex and Q. coccifera, 28 Jun. 2016, leg. L. Friedman (SMNHTAU); 8 ex., Kefalonia, SW Neochori, Kalos Mts, Demotokitos Mt, 38¹9.660’ N, 20³6.674' E, 400-600 m, on Quercus, 29 Jun. 2016, leg. L. Friedman (SMNHTAU); 6 ex., Kefalonia, Komitata, $38^{\circ} 21.149^{\prime}$ N $20^{\circ} 35.863^{\prime} \mathrm{E}, 550 \mathrm{~m}$, on Quercus ilex and Q. coccifera, 29 Jun. 2016, leg. L. Friedman (SMNHTAU); 2 ex., Kefalonia, Agia Dinata Mts, $5 \mathrm{~km}$ N of Dilinata, $38^{\circ} 16.013^{\prime} \mathrm{N}, 20^{\circ} 30.712^{\prime}$ E, $700 \mathrm{~m}$, on Quercus ithaburensis macrolepis, 30 Jun. 2016, leg. L. Friedman (SMNHTAU); 1 đ̃, Epirus, Velia, Pindos, Grevena, 8 Aug. 1986, leg. Barries and Cate (SDEI); 1 § Kreta ins. Dikti Mts 5 Jun. 2001, Lesithi plateau, $800 \mathrm{~m}$, leg. J. Voříšek (BMNH).

SYRIA: 2 $ぇ ð$, Syria, Reitter (BMNH); 1 q, Syr[ia], coll. A. Rätzer (NMBE).

TURKEY: 1 วิ, Asia min., Adana, Reitter (BMNH); 1 ô, centr. Ankara N, $10 \mathrm{~km} \mathrm{~S}$ of Ilgaz, $18 \mathrm{Jul}$.

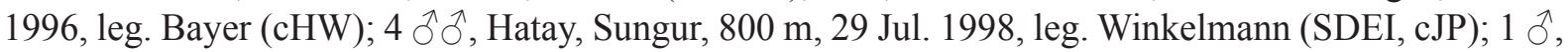
Asia Minor, Smyrna prov., Bozdagh Mts, Goelchik, 29 Jul. 1931, leg. Uvarov (BMNH); 1 , , Bozdag nr. Izmir, 26 Jun. 1971, leg. Lodos (BMNH); 4 ổ, Bursa, Cagliyan env., 10-14 Jul. 1997, leg. Prudek and Riha (cHW); 17 ex., Anat. M., Nur Daglari, Hasanbeyli, 1150 m, 16 Jul. 1996, Tymer and Voř́šek leg. (cRB, cJP); 1 ð̊, Icel (Mersin NW), Findikpinari, 1000 m, 26 Jul. 1998, leg. Winkelmann (cHW); 11 ex., Ankara N, Ilgaz S Korgun, N, 18 Jul. 1996, leg. Bayer and Winkelmann (SDEI, cHW); 1 ex., Hop pass, $15 \mathrm{~km}$ NE Mardin, 37²3" N, 4051" E, 16 May 2001, leg. Kadlec (cRB); 1 ō, Tosya, Ene, Ada Dagi N,

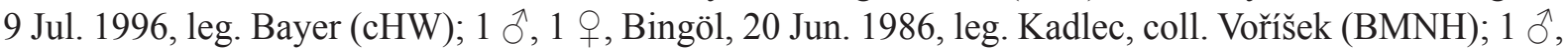
Olüdenis, Fethyie env., 8 May 1991, leg. Zbuzek (cRB); 2 ex., Ankara N, Tosya N, Karadere, 16 Jul. 1996, leg. Bayer and Winkelmann (cHW); 1 Ő, 1 ㅇ, Van prov., Alacabük Dagi, Kuskuh Kiran, Göründü, 
28 Jun.-2 Jul. 1997, D. Hauck leg. (cHW); 1 ふै, Avsallar, b. Incekum, 22 km W of Alanya, 9-23 May

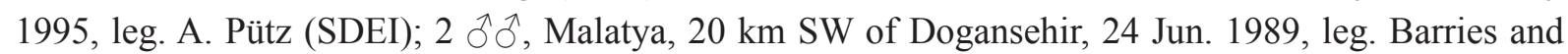

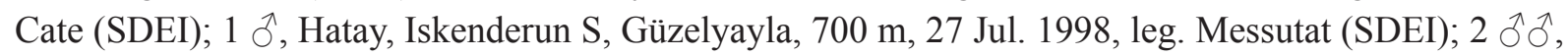
Nur Daglari, Husanbeyli, 1150 m, 16 Jul. 1996, leg. Tyrner and Voříšek (BMNH); 1 ex., Göynük NNW

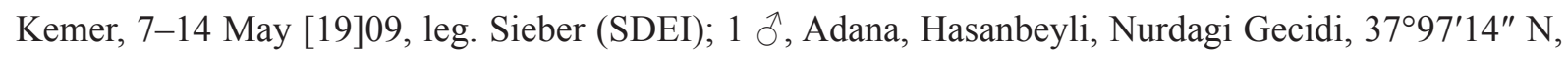
36³4'30" E, 800-1100 m, 24 May 2002, leg. Skale (SDEI); 1 §, Tarpus [?], 1200 m, leg. S. Lundberg (SDEI); 1 §ૈ, Anatol. Centr. Tal zw. Tünceli u. Ovacik, 1200 [m], 20 Jul. 1985, leg. W. Heinz (SDEI); 32 ex., Ankara N, 10km S of Ilgaz, 18 Jul. 1996, leg. Bayer (cHW, cCG, NMBE); 1 §, 1 \%, Destigin, 5

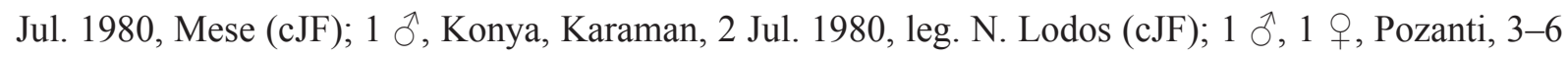
Jul. 1983, leg. Jiri Hladil (cJF).

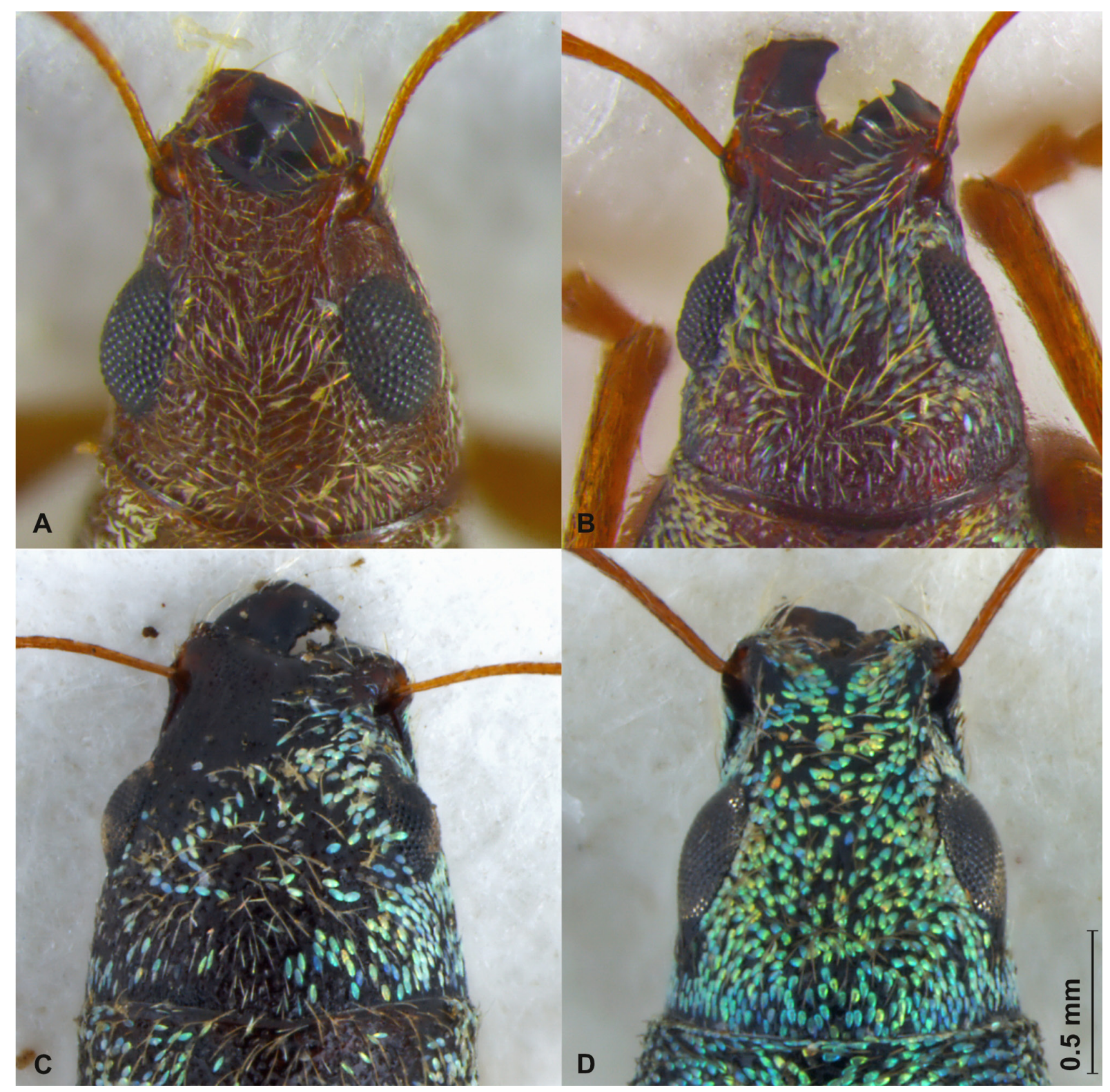

Fig. 5. A-D. Heads of species of Polydrusus (Conocetus). A. Polydrusus grandiceps Desbrochers des Loges, 1875, male. B. Polydrusus transjordanus sp. nov., male. C. P. stierlini Schilsky, 1910, female. D. P. angustus (Lucas, 1854), male. 


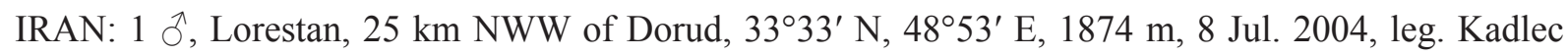
(cRB); 1 §̂, Lorestan, 25 km NWW of Dorud, 1874 [m], 8 Jul. 2004, leg. Rejek (cHW).

\section{Comments on new synonymies}

\section{Polydrusus bardus $=P$. gracilicornis Kiesenwetter, 1864 syn. nov.}

Polydrusus gracilicornis was described based on specimens from Crete (Greece), as Kiesenwetter wrote: "Von Zebe auf Creta gesammelt (Ksw.), von Kraatz mir auch, als in Griechenland gesammelt, mitgetheilt." [Collected by Zebe on Crete (Ksw.; thus referring to his own collection)...]. Indeed Kraatz only told Kiesenwetter that the (new species) was also found by him in Greece (= mainland Greece, supposedly). There is a single specimen in the Kiesenwetter collection at the ZSM, which is here designated as lectotype of Polydrusus (Conocetus) gracilicornis Kiesenwetter, 1864 with the following label data: Creta // gracilicornis Candie Ksw // Kiesenwetter // [red label:] Lectotype Polydrusus (Conocetus) gracilicornis Kiesenwetter, 1864 des. C. Germann 2016.

\section{Polydrusus bardus $=P$. cylindrithorax Desbrochers des Loges, 1900 syn. nov.}

The description of $P$. cylindrithorax was based on specimens from Rhodes. All examined specimens from Rhodes belong to $P$. bardus.

\section{Polydrusus bardus $=$ P. quadraticollis Desbrochers des Loges, 1902 syn. nov.}

Based on examined specimens of $P$. quadraticollis from Smyrne (= Izmir). All examined specimens from Turkey belong to $P$. bardus.

\section{Remarks}

As already stated by Schilsky (1910), P. (Conocetus) bardus was often confused and misinterpreted (see under $P$. crinipes sp. nov.), and it was only by examining the type specimen that the true identity of the species could be clearly established. Desbrochers des Loges (1902) confused it with P. marcidus in his redescription, but there are also examples in the revised collections where P. bardus (or P. crinipes sp. nov.) were confused with P. calabricus (e.g., det. Stöcklein NMB).

\section{Variability}

This often misinterpreted species varies considerably in size, vestiture (scale size, colour), vestiture of legs (metallic hairs with or without scales intermixed), length and orientation of hairs on elytra and, to a lesser degree, in the shape of the pronotum, which is mirrored in the different names aiming for the shape of the pronotum (cylindrithorax, quadraticollis). However, careful examination of the shape of the penis as well as the internal sac across a comprehensive body of material (see above) allowed the identification of a single, more widely distributed species.

\section{Ecology}

Desbrochers des Loges (1902) indicated "sur les Pins" [on pine trees] in his description. On Crete Polydrusus bardus was beaten from Quercus ithaburensis (C. Germann, pers. obs.) and Q. pubescens (Thompson, data taken from labels). Furthermore, according to label data from various collectors, the species was also collected from Castanea sativa in Turkey (e.g., N. Lodos), and from Quercus ilex, Q. coccifera and Q. ithaburensis macrolepis in Greece (L. Friedman). Records are from April to August, from sea level to subalpine altitudes. 


\section{Polydrusus (Conocetus) baudii (Faust, 1889)}

Figs 4E-F, 8D, 9N, 11

\section{Material examined}

CYPRUS: 3 ふึ่, 1 ๆ, Plantania Forest Station, 1370 m, 26 Apr. 1945, leg. G.A. Mavromoustakis

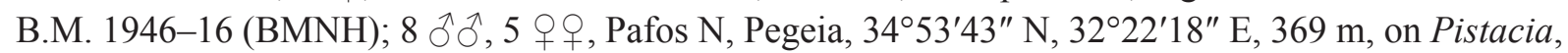
Pinus, Juniperus and Cistus, 17 Apr. 2010, leg. Behne (18) (SDEI); 2 $\partial^{\lambda}, 20 \mathrm{~km}$ NW of Pafos Pegaia,

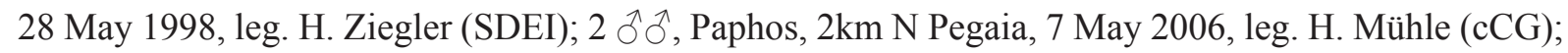

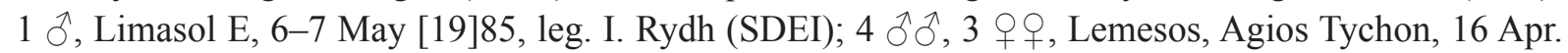
[20]13, leg. J. Messutat (cCG, cJM); 4 $\widehat{\jmath}, 2$ 우으, Paphos Anarita, 23 Apr. [20]10, leg. J. Messutat

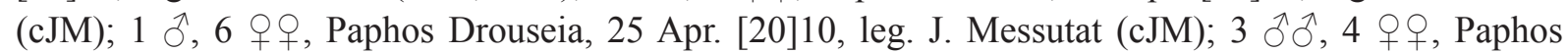
Polis, 22 Apr. [20]10, leg. J. Messutat (cJM); 1 q, Larnaka, 3 km S of Melini, 3 May 2006, leg. Mühle (cCG); 1 q, Besika Bay, 1927, leg. G.C. Champion B.M (BMNH); 2 + + , Skarinou, 16-18 Jun. 1993, leg. Krajcik (cRB); 1 q, Paphos, Kidhasi, lit du Dhiarizos, 11 May 2008, leg. G. Alziar (cJP); 1 q, Lapittias ... [illegible], Apr. [19]53, leg. Th. Shiakides (BMNH); 1 q, Kritou Tera, 12 km südl. von Polis, Olivenhain, 2 May, leg. W. Apfel (NMEG); 7 ex., Troodos, Caledonia Falls, Krios River, 1 km N Pano Platres, 1600 m, 10 Aug. 2005, leg. L. Friedman (cPB); 1 ex., Troodos Mesopotamos, Krios River, picnic area, 11 Aug. 2005, leg. L. Friedman and Y. Antoniou (cPB).

\section{Type locality}

Cyprus.

\section{Variability}

Rather constant in size and vestiture, the colour of the scales varies from greenish to brownish.

\section{Ecology}

Collected from various trees (L. Behne; Pistacia, Pinus, Juniperus, Cistus) in creek valleys and Olive groves at various altitudes in spring and summer (from April to August).

Polydrusus (Conocetus) calabricus (Faust, 1890)

Figs 3E-F, 7G, 9I-J, 12

\section{Material examined}

GREECE: 2 ふふ, Peloponesos b. Clokos Mts, 2 km NE Petsaki, 900-1000 m, 20 May 2004, J. Voříšsek

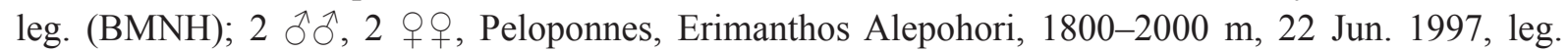
Winkelmann (SDEI); 2 ex., Epirus, Umgeb. Konitsa, 9 Jul. 2006, leg. W. Ziegler (cHW); 1 §, Corfu N, Mt Pandokratoras, Lafki (3 km SE Acharavi), 3946'24" N, 1950'41" E, 420 m, 21 May 2012, leg. Bahr

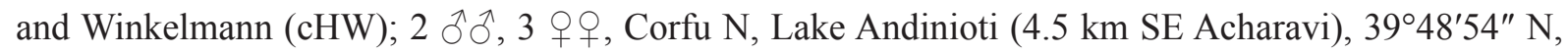
1951'42" E, 22 May 2012, leg. Bahr and Winkelmann (cCG, cHW); 1 đ̃, 1 q, Pelopones, coll. Apfelbeck

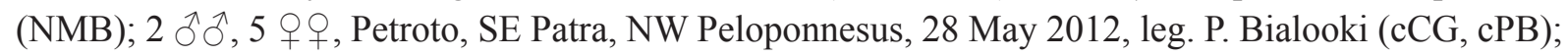
1 ภ, 1 \&, Kefallonia, Poros env., 29 May-5 Jun. 1997, leg. Ing. K. Schön (cRB); 1 o, Peloponnes 10 km sü Kalamata über Ano Ve.g., ca. 400 m 1 May 1990, leg. A. Elbert (SDEI); 1 \&, Peloponnes $3 \mathrm{~km}$ sü Kalamata Schluchteingang [entrance of a gorge], ca $150 \mathrm{~m}, 1$ May 1990, leg. A. Elbert (SDEI); 1 Oे, Epirus Nomos Thesprotia, Tal zw. Margariti und Morti, 24 May 1992, leg. A. Elbert (SDEI); 1 ô, Kalavrita, 23 May 1990, leg. Svec (cRB); 2 ex., [Peloponnese] Ilias, Panopoulos, Eichenwald NW, 21.39 E, 37.50 N, 30 May 2006, leg. Rieger (cCG, cJM). 
ITALY: 2 ex., Capo Leuca, 25 May 1948, leg. Giordani-Busulini (cJP); 1 ex., Puglie, S. Menaio, Jun. 1965, leg. Abbruzzese (cJP); 1 ex., Aquila, leg. Reitter, coll. Marshall (BMNH); 1 q, Puglia, Circ. Mar. Piccolo (TA), 5 Jun. 1977, leg. Montemurro (NMB); 1 §̂, Puglia, Francavilla F. (BR), 140 m, 15 May 1976, leg. F. Angelini (cJM).

\section{Type locality}

Calabria (Italy).

\section{Variability and similar species}

The diagnosis given by Faust (1890) is precise: “...sie kann sehr leicht mit angustus Luc. verwechselt werden, wenn man die breitere Stirn, die kürzeren, mehr gewölbten Augen, den breiten Rüssel und die

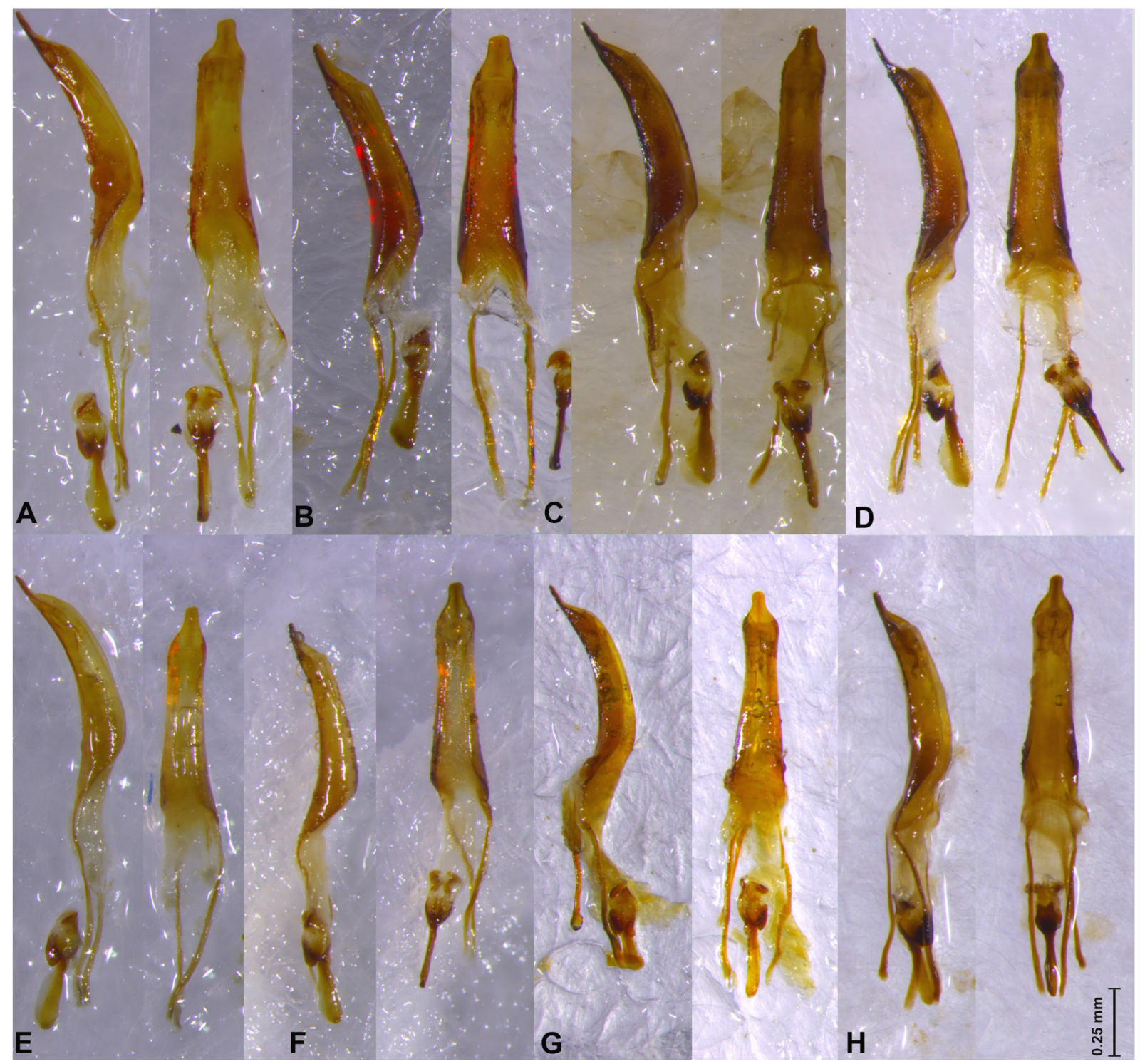

Fig. 6. A-D. Penis of Polydrusus (Conocetus) grandiceps (Desbrochers des Loges, 1875), lateral and dorsal views. A. Lectotype: Lebanon. B. Turkey, Erdemli. C. Israel, Nimrod. D. Israel, Panyas. E-H. Penis of P. transjordanus sp. nov., lateral and dorsal views. E. Jordan, Ajlun. F. Israel, Nahal Oren. G. Israel, Mi'ilya. H. Holotype: Israel, Mt Meron. 
dünneren Beine von calabricus nicht beachtet." [...it (P. calabricus) can very easily be confused with angustus, if the wider frons, the shorter and more convex eyes, the wider rostrum and the more slender legs of calabricus were not considered]. See also the comments above under $P$. angustus, the most similar species to $P$. calabricus.

\section{Ecology}

Collected from May to June, from sea level to subalpine altitudes.

Polydrusus (Conocetus) festae (F. Solari, 1925)

Figs 1C-D, 7B, 9D, 12

\section{Material examined}

GREECE: 1 q, [Crete,] Suda Bay, leg. G.C. Champion B.M. 1927-409 (BMNH); 8 $\widehat{\partial}, 6$ q , GR

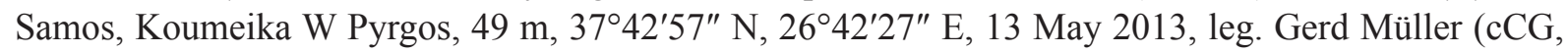

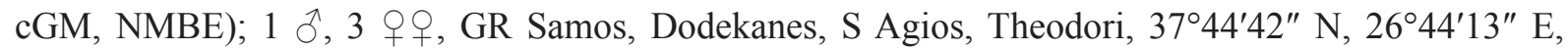
296 m, 11 May 2013, leg. Gerd Müller (cGM, NMBE); 1 ㄱ, 2 우우, GR, Samos, Oros Kerkis Mt,

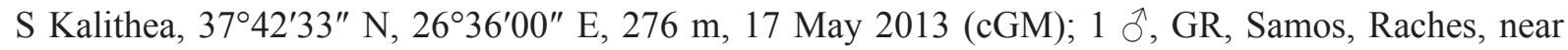

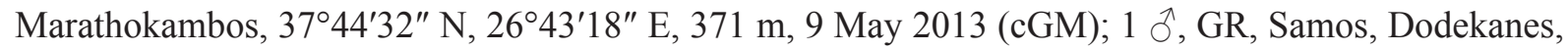

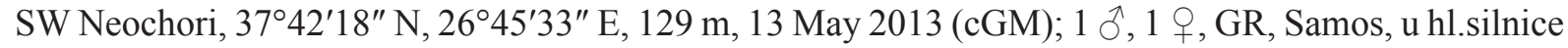
Karlovasi-Pythagorio (u odb. Na Koumeiku), 16 Jun. 2007, leg. Ing. K. Schön (cRB); 1 đ̊, Samos Isl.,

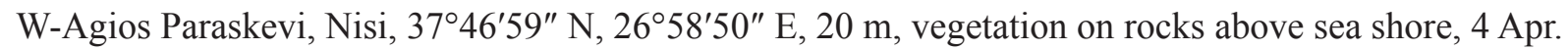
2010, leg. C. Germann (cCG); 1 ô, Kr. Omalos, leg. S. Lundberg (SDEI); 1 , , GR-Samos, Votsalakia env., 11-20 Jun. 2007, leg. Ing. K. Schön (cRB); 1 q, Kreta, leg. Paganetti, coll. Jörger (NMB).

LYBIA: 1 q, Cirene U.C., Apr. [19]38, leg. G. Frey (NMB).

\section{Type locality}

LIBYA: Cyrenaica.

\section{Remarks}

Solari (1925) provided the following (type) localities (included in Fig. 12): Derna, Zavia (=Az Zawiyah), Mechili, Tecnis (= Taknis) and Tolmetta. The first records of $P$. festae outside of Libya were already reported by Germann et al. (2015) as specimens of an unknown Conocetus sp. from Samos Island. It is remarkable that this brachypterous and thus supposedly little-mobile species occurs across the Mediterranean Sea. This can be explained by the recent introduction of this species in Greece (Samos Island and Crete), although the first specimens known from Crete (without collecting date, see above) were collected by Gustav Paganetti-Hummler (1871-1949), hence a very recent introduction can be excluded. Polydrusus festae is therefore recorded here for the first time from Greece.

\section{Variability and similar species}

The species was compared with both P. baudii and P. marcidus by Solari (1925), based on the key by Schilsky (1910). The squamulate scutellum, the head shape, pronotum and the oval elytra (Fig. 1C-D) as well as its brachyptery are very characteristic and not shared with other species of the subgenus.

\section{Ecology}

Collected from February (Solari 1925) to June, from the sea shore to colline altitudes. Found on flowering Quercus ilex bushes (G \& U. Müller and C. Germann; photograph of the habitat in Germann et al. 2015). 
Polydrusus (Conocetus) grandiceps (Desbrochers des Loges, 1875)

Figs 2A-B, 6A-D, 9P, 11

\section{Material examined}

\section{Lectotype}

The following syntype specimen is hereby designated as lectotype of Polydrusus (Conocetus) grandiceps:

LEBANON: 1 $\widehat{\text {, }}$, Conocetus grandiceps Desbr. Libanon cèdres Desbroch. [handwritten] // Syntypus [printed] // Faust vid. teste // Sch. [green paper] // coll. L. v. Heyden [printed] // [red label:] Lectotype Polydrusus. (Conocetus) grandiceps (Desbrochers des Loges, 1875) des. C. Germann 2016 (SDEI).

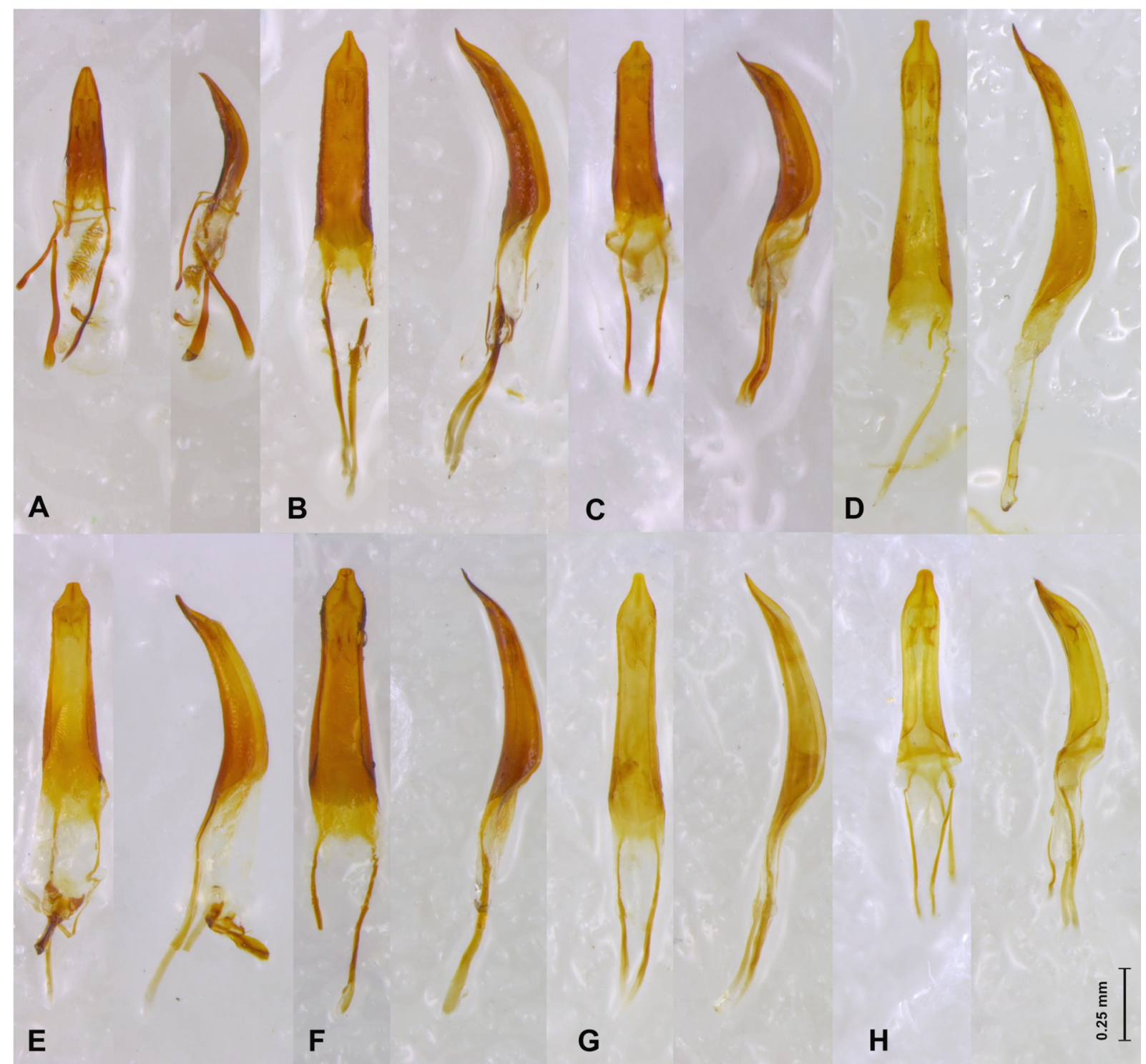

Fig. 7. A-H. Penis of Polydrusus (Denticonocetus) and P. (Conocetus) spp., dorsal and lateral views. A. P. (D.) kahri Kirsch, 1865, Italy, Sicily. B. P. festae (F. Solari, 1925), Greece, Samos. C. P. stierlini (Schilsky, 1910), Greece, Macedonia. D. P. rhodiacus (Schilsky, 1912), Cyprus, Dionios. E. P. crinipes sp. nov., Greece, Corfu. F. P. angustus (Lucas, 1854), Greece, Crete. G. P. calabricus (Faust, 1890), Greece, Peloponnese. H. P. bardus Gyllenhal, 1834, Greece, Crete. 


\section{Paralectotype}

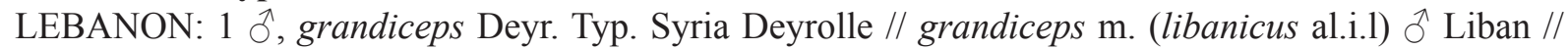
Syntypus [red label] // coll. L. v. Heyden // [red label:] Paralectotype Polydrusus (Conocetus) grandiceps (Desbrochers des Loges, 1875) des. C. Germann 2016 (SDEI).

\section{Other material examined}

EGYPT: $2 \hat{\jmath}, 1$, , Cairo Aegypt., coll. Frey (NMB).

ISRAEL: 1 ex., Qiryat Tiv'on, on Quercus ithaburensis, 25 Apr. 1954, coll. M. Sternlicht (BMNH);

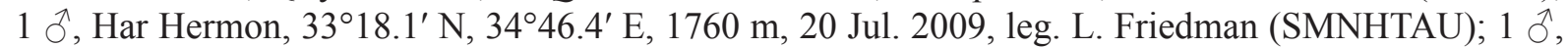

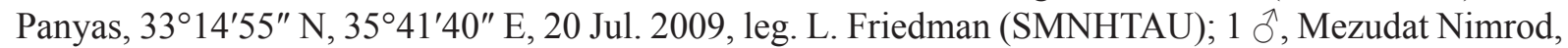
27 May 1999, leg. V. Chikatunov (SMNHTAU); 1 ๙ิ, Mezudat Nimrod, 27 May 1999, leg. L. Friedman (SMNHTAU); 2 ex., Har Hermon, 1600 m, 5 Aug. 2004, leg. L. Friedman/A. Freidberg (SMNHTAU); 4 ex., Har Hermon, $33^{\circ} 18^{\prime}$ N, 35 $46^{\prime}$ E, $1500-1600$ m, 6 Jun. 2002, leg. L. Friedman/A. Freidberg (SMNHTAU). 1 ex., Har Hermon, 1600 m, 12 Jun. 2003, leg. L. Friedman/A. Freidberg (SMNHTAU);

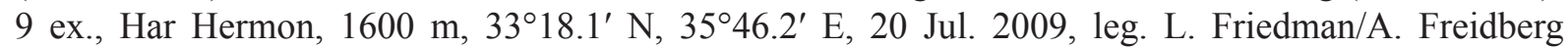

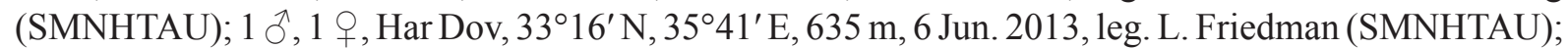
$1 \hat{\jmath}$, Hermon Field School, 18 May 2009, leg. L. Friedman (SMNHTAU); 1 , Har Dov, Mizpe Ramta, 16 Jun. 1999, leg. L. Friedman (SMNHTAU); 1 ô, Panyas, 14 Jun. 1996, leg. A. Freidberg (SMNHTAU); 1 q, Har Dov, 33¹5' N, 3540' E, 300 m, 6 Jun. 2013, leg. L. Friedman (SMNHTAU).

JORDAN: 1 § , $5 \mathrm{~km}$ NE of Ajlun, 32.36754 N, 35.77937 E, 1005 m, 10 Jun. 2011, leg. C. Monnerat

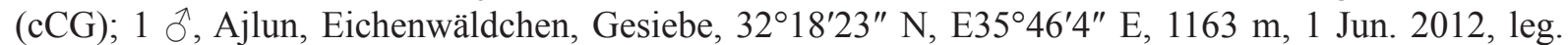
M. Borer (cCG); 1 §, 3 ex., Amman, 800 m, 27 Jun. 1957, leg. J. Klapperich (SDEI); 1 \& Dehbeen b. Jerash, 19 Jun. 1959, leg. J. Klapperich (SDEI); 3 ex., Umgb. Jerash, 31 Aug. 1968, leg. J. Klapperich (SDEI).

LEBANON: 1 ð, Mt Liban, coll. D. Sharp (BMNH); 1 ex., Beyrouth (Beirut), coll. J. Pelletier (cJP); 1 ex., Achkout, coll. J. Pelletier (cJP); 1 đ’, Northern gov., 7 km NE of Batroun, Qnat, $34^{\circ} 15.323^{\prime}$ N, 35 53.667' E, 1195 m, macchia, swept and singled, 18 Jun. 2016, leg. A. Kotán, P. Nemes and T. Németh

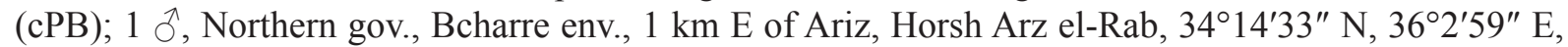
1900 m, ancient Cedrus forest, swept and singled, 20 Jun. 2016, leg. A. Kotán, P. Nemes and T. Németh

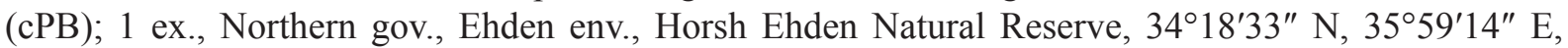
1525 m, swept and singled, 19 Jun. 2016, leg. A. Kotán, P. Nemes and T. Németh (cPB); 1 त̂, 1 क, Northern gov., Tannourine env., $2 \mathrm{~km} \mathrm{~N}$ of Harissa, Tannourine Cedars Nat. Reserve, $34^{\circ} 12^{\prime} 34^{\prime \prime} \mathrm{N}$, $35^{\circ} 55^{\prime} 45^{\prime \prime}$ E, 1750 m, swept and singled, 18 Jun. 2016, leg. A. Kotán, P. Nemes and T. Németh (cPB).

SYRIA: 1 đૈ, 6 ex., Burqush env., NW Damascus, 25 May 1998, leg. S. Kadlec (cRB); 1 ô, Syria (Tamaze env.), Jabal al-Ansariyah, E slope, 6 Jun. 1999, leg. S. Kadlec (cRB).

TURKEY: 2 đ̊ đ, Anatol. m., Toros Daglari, 900 m, 6 Jun. 1991, leg. S. Kadlec, coll. Voříšek (BMNH); 3 ex., Icel (Erdemli NW), Aydinlar SW, 25 Jul. 1998, 1000 m, leg. H. Winkelmann (HW); 6 ex., Icel (Erdemli NW), Aydinlar SW, 1000 m, 25 Jul. 1998, leg. Messutat (cJM); 1 ex., Aydinlar b. Erdemli,

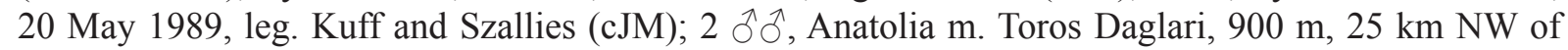
Erdemli, 6 Jun. 1991, S. Kadlec leg. (BMNH).

\section{Comments on new synonymy}

Polydrusus grandiceps $=$ P. zurcheri (Schilsky, 1912) syn. nov

The following syntype specimen, fully in accordance with the locality given in the original description (Hadjin-Dagh [= Saimbeyli in Turkey, Adana]), is hereby designated as lectotype of $P$. (Conocetus) zurcheri: 
TURKEY: 1 Oे, Hadjin Dagh As. minor // 6 // Ch. Zurcher // Zurcheri * Schils. // Lectotype Polydrusus (Conocetus) zurcheri (Schilsky, 1912) des. C. Germann 2016 (MFN).

\section{Variability}

Specimens vary from dark brownish with scarce and spotty scales to light brown with denser scales. Freshly emerged specimens (where the mandibular scars are still present; these quickly get lost) show denser standing scales.

\section{Ecology}

Collected on or nearby Quercus ithaburensis and Quercus sp. in general (sifted from leaf litter; M. Borer oral comm.), but also from Cedrus sp. (Cupressaceae). Lives sympatrically in similar habitats with P. transjordanus sp. nov. Collected from April to July, from sea level up to high montane altitudes.

Polydrusus (Conocetus) marcidus Kiesenwetter, 1864

Figs 4C-D, 8C, 9M, 10

\section{Material examined}

\section{Type material}

Two syntypes of Polydrusus marcidus Kiesenwetter, 1864, where the first one is selected as lectotype:

GREECE: 1 \%, Taygetos // [red label:] Lectotype Polydrusus (Conocetus) marcidus Kiesenwetter, 1864 labelled C. Germann 2016 (ZSM); 1 , , [without label, but mounted in exactly the same way as the other specimen, with new label printed on red paper:] Paralectotype Polydrusus (Conocetus) marcidus Kiesenwetter, 1864 labelled C. Germann 2016 (ZSM). The locality is handwritten, supposedly by Kiesenwetter himself. The exact locality was not indicated in the original description; Kiesenwetter (1864: 254) wrote very generally as usual at that time: "Aus Griechenland (Krüper)..." [from Greece (Krüper)]. He furthermore mentioned: "...von Kraatz mitgetheilt [reported by Kraatz]." However, the chosen lectotype and paralectotype, which were the only specimens pinned under the species' name in the collection of Ernst August Hellmuth von Kiesenwetter (in ZSM), fit perfectly the author's description and it is very likely that the author based his description on them. Kiesenwetter probably bought them from Theobald Krüper, who was known to sell insects from Greece from 1859 (Horn et al. 1990), then labelled them himself.

Four syntypes of Polydrusus gracilis (Stierlin, 1888) where the lectotype and three paralectotypes are selected in accordance with the description by Stierlin (1888b):

GREECE: 2 우 [on the same pin, the upper one is selected here as lectotype], gracilis Strl Graecia Zeiller [unknown handwriting] // Db. // gracilis $9 \hat{\jmath} / /$ [ [both are females] (collection L. von Heyden,

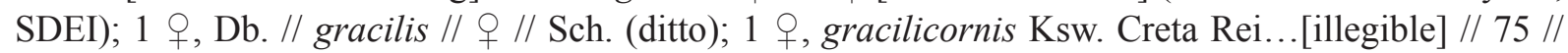
Db. // gracilis // q Sch.(ditto). // [red label:] Lectotype/Paralectotypes Polydrusus (Conocetus) gracilis (Stierlin, 1888) (= marcidus Kiesenwetter, 1864) labelled C. Germann 2016. The lectotype and all syntypes belong to $P$. marcidus; therefore the synonymy of $P$. gracilis with $P$. marcidus can be approved here.

\section{Type locality}

GREECE: Peloponnese, Taygetos.

\section{Other material examined}

ALBANIA: 1 †, Tepelenë Pass N Buz, 900 m, 10 Jul. 2010, leg. H. Mühle 55 (cCG). 
BULGARIA: 1 q, near Ju Rodopi, Jez Kŭrdzhali , 9 Aug. 1986, leg. R. Borovec (cRB); 1 q, Pirin, Sugarevo env., 700-900 m, leg. R. Borovec (cRB); 1 क , Delschewo [Delschevo], Pirin, auf Trockenwiesen [on dry meadows], 24 Jul. 1989, leg. F. Burger (SDEI).

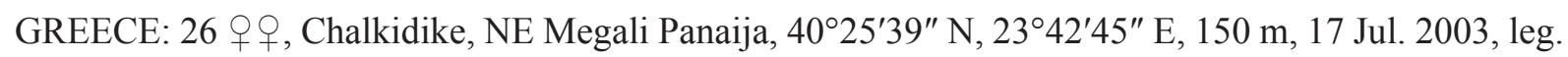
Bayer (cCG, cHW, NMBE); 1 q, Kreta, ins. Dikti Mts, Leshiti plateau, 800 m, 5 Jun. 2001, leg. J. Vořŕšek (BMNH); 2 우, Ioanina, Elati, 7 Jul. 2003, C. Cocqempot leg. (cJP); 1 q, Crete SW, Paleochora NE,

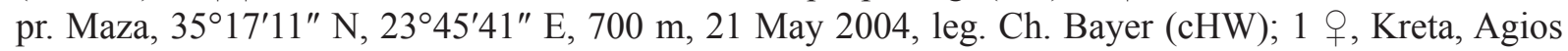
Nikolao, 24 Apr. 1971, leg. W. Wittmer (NMB); 1 \&, Katerini, Ag. Dimitrios, 800 m, 19-21 Jul. 1990, leg. Schuh and Zabransky (SDEI); 3 우, Makedonia, Chalkidike, $3 \mathrm{~km}$ NE of Megali Panaija, 17 Jul. 2003, leg. F. Bahr (cHW); 2 q , Agios Grangatiko [Grammatiko], Prov. Pella, SW Edessa, 31 Jul. 2009 leg. W. Ziegler (cHW); 1 + , Voria Pindos, Morti Trockenhang [dry slope], 25 Jun. 1997, leg. Winkelmann (cHW); 1 q, Pella, Asrogogia, 29 Jul. 2009, leg. W. Ziegler (cHW); 1 q, Kreta, Prina Kalamafka, 7 May 1999, leg. K. Liebenow (SDEI); 2 우, Korfu, Liapades, 20 Jun. 2001, leg. K. Liebenow (SDEI); 1 +, Korfu, Sidari, 1 Aug.1989, leg. W. Suppantschitsch (SDEI); 1 q, Korfu, Lipades, 30 Jul. 1989, leg. W. Suppantschitsch (SDEI); 1 \%, Epirus, Velia, Pindos nr. Grevina, 8 Aug. 1996, leg. Barries and Cate (SDEI); 1 \%, Samos, May 1987, leg. Zimmermann (SDEI); 1 q, Parnass, leg. Paganetti (NMB); 1 q, KR [Crete], Chania, Kuma-See, 4 Jun. 1994, leg. G. and M. Novak (SDEI); 1 q, Kreta, Omalos, 25 Jul. 2009, leg. M. Egger (cCG); 1 + , Kreta, Kera Izermiadon, 30 Jun. [19]82, leg. Bernhauer (coll. JM); 1 q, Thesprotia, Plataria b. Igoumenitsa, Jun. 1994, leg. J. Frank (cJM); 1 , , Voria, Pindos E, Grevena NW, Morfi, 25 Jun. 1997, leg. Messutat (cJM); 17 q , Kefalonia, Agios Eleftherios, 3 km NW, $38^{\circ} 11.482^{\prime} \mathrm{N}, 20^{\circ} 35.658^{\prime} \mathrm{E}, 670 \mathrm{~m}$, on Quercus ilex and Q. coccifera, 27 Jun. 2016, leg. L. Friedman (SMNHTAU); 26 우, Kefalonia, Tzanata, Mycenean Tholos Tomb, 3808.509' N, 2045.646' E, 70 m, on Quercus ilex and Q. coccifera, 28 Jun. 2016, leg. L. Friedman (SMNHTAU); 4 우, Kefalonia,

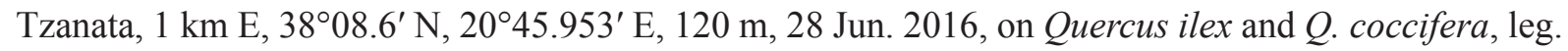
L. Friedman (SMNHTAU); 4 우, Kefalonia, SW Neochori, Kalos Mts, Demotokitos Mt, 38¹9.660' N, $20^{\circ} 36.674^{\prime}$ E, 400-600 m, on Quercus, 29 Jun. 2016, leg. L. Friedman (SMNHTAU); 4 q, , Kefalonia, NW Komitata, Kalos Mts, Vathoula Mt, 38 $21.484^{\prime}$ N, 20 $35.145^{\prime}$ E, 640-730 m, on Quercus ilex and Q. coccifera, 29 Jun. 2016, leg. L. Friedman (SMNHTAU); 11 우, Kefalonia, Charakti, 38¹0.310' N,

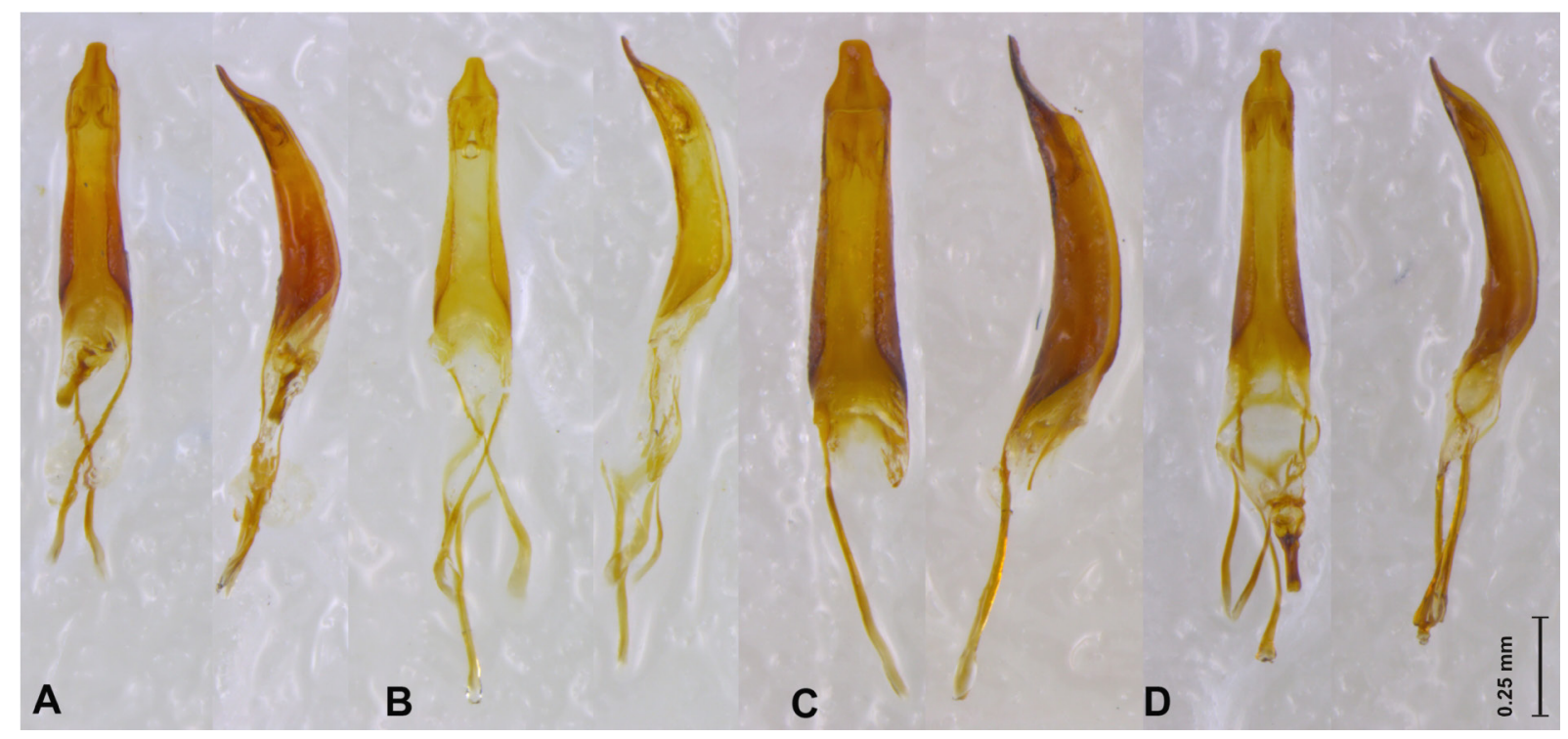

Fig. 8. A-E. Penis of Polydrusus (Conocetus) spp., dorsal and lateral views. A. P. bardus Gyllenhal, 1834, Turkey, Hatay. B. P. bardus, Iran, Lorestan. C. P. marcidus Kiesenwetter, 1864, Turkey, Antalya. D. P. baudii (Faust, 1889), Cyprus, Lemesos. 


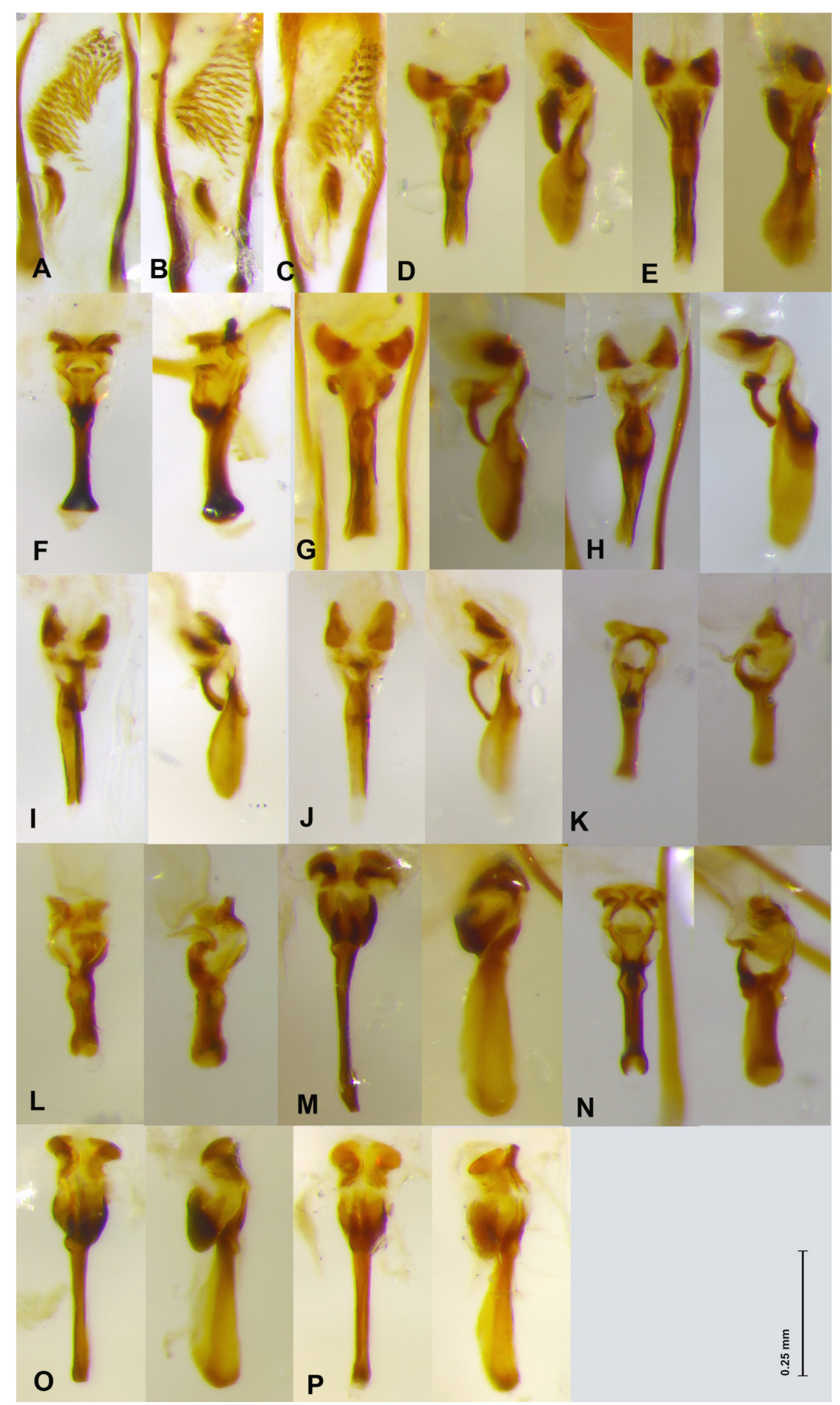

Fig. 9. A-C. Internal sac of Polydrusus (Denticonocetus) kahri Kirsch, 1865. A. Italy, Sicilia. B. Croatia, Istria. C. Italy, Monte Baldo. D-P. Internal sac, dorsal and lateral views of: D. P. (Conocetus) festae (F. Solari, 1925), Greece, Samos. E. P. stierlini (Schilsky, 1910), Greece, Poligiros. F. P. rhodiacus (Schilsky, 1912), Cyprus, Dionios. G. P. crinipes sp. nov., Greece, Corfu. H. P. angustus (Lucas, 1854), Greece, Crete. I. P. calabricus (Faust, 1890), Greece, Patra. J. Ditto, Italy, Capo Leuca. K. P. bardus Gyllenhal, 1834, Greece, Crete. L. Ditto Iran, Lorestan. M. P. marcidus Kiesenwetter, 1864, Turkey, Antalya. N. P. baudii (Faust, 1889), Cyprus. O. P. transjordanus sp. nov., Jordania, Ajlun. P. P. grandiceps (Desbrochers des Loges, 1875), lectotype, Lebanon. 
$20^{\circ} 41.359^{\prime}$ E, 530 m, on Quercus ilex and Q. coccifera, 28 Jun. 2016, leg. L. Friedman (SMNHTAU); 7 우, Kefalonia, Komitata, $38^{\circ} 21.149^{\prime}$ N, $20^{\circ} 35.863^{\prime}$ E, 550 m, on Quercus ilex and Q. coccifera, 29 Jun. 2016, leg. L. Friedman (SMNHTAU); 4 우, Kefalonia, Agia, Dinata Mts, 5km N Dilinata, $38^{\circ} 16.013^{\prime} \mathrm{N}, 20^{\circ} 30.712^{\prime} \mathrm{E}, 700 \mathrm{~m}$, on Quercus ithaburensis macrolepis, 30 Jun. 2016, leg. L. Friedman (SMNHTAU).

TURKEY: 2 우, Bursa, Cagliyan env., 10-14 Jul. 1997, P. Prudek and M. Riha leg. (cHW); 2 우, Eskisehir Sakari Ilica, near Gümele, 6-9 Jul. 1997, leg. P. Prudek and M. Riha (cHW); 1 +, Myra, 5 May 1991, leg. B. Zbuzek (cRB); 1 +, Myra 5 May 1991, 150 km SW of Antalya, leg. Zd. Jindra (cRB); 1 , $90 \mathrm{~km}$ S of Antalya, Kumluca, 22 May 1991, leg. W. Suppantschitsch (SDEI); 2 q, , Ankara N, Ilgaz S, Korgun N, 18 Jul. 1996, leg. H. Winkelmann (cHW); 1 Ĵ, 1 q, Kemer Prov., Antalya, 3-15 Jul. 1989, leg. H. Schmid (SDEI); 1 + , Kestebek, 24 Jun. 1991, Kudrna leg. (BMNH); 1 + , Antalya, Kemer, 31 May 1991, G. Gillerfors (SDEI); 1 ô, Antalya, Kemer, 31 May 1991, leg. G. Gillerfors (SDEI); 1 ふै, Prov. Antalya, Beldibi, 7 Jul. 1992, leg. H. Schmid (SDEI); 1 ô, Prov. Antalya, Kemer, 3 Jun.

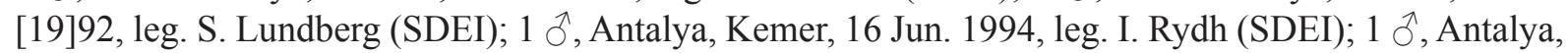
Kemer, 1600 m, 20 May [19]91, leg. W. Suppantschitsch (SDEI); 1 +, Zonduldak, Karabük, 20 km E, 22-23 Jun. 1996, leg. V. Svihla (cRB).

\section{Remarks and variability}

Polydrusus marcidus seems to reproduce parthenogenetically in the western part of the distribution area. Male specimens are presently only recorded from Turkey (Antalya). Males differ considerably from females (bigger and more stout pronotum, fore tibiae directed inwards towards apex, generally bigger in size; Fig. 4C-D). Some females from the amphigonic populations (Antalya, Kestebek, Myra) are considerably bigger than other populations.

\section{Ecology}

Found on two occasions on dry slopes or meadows, once in Bulgaria (F. Burger) and in Greece (H. Winkelmann). From label data the following trees are mentioned: Quercus coccifera, Q. ilex and Q. ithaburensis macrolepis (by L. Friedman from Greece). Collected from April to August, at hill to (high-) montane altitudes.

\section{Polydrusus (Conocetus) rhodiacus (Schilsky, 1912)}

Figs 2E-F, 7D, 9F, 12

\section{Material examined}

Type material

One syntype specimen, which is selected here as lectotype:

CYPRUS: + , Chypre [printed] // Ch. Zürcher [handwritten] // rhodiacus m. [mihi] ô $q$ [handwritten by Friedrich Julius Schilsky on bluish paper] // [the following label of red paper was added:] Lectotype Polydrusus (Conocetus) rhodiacus (Schilsky, 1912) labelled C. Germann 2016 (MFN).

\section{Type locality \\ CYPRUS.}

Other material examined

CYPRUS: 1 ðૈ, Dionos (Chypre), 28 May 1974, coll. Voříšek (BMNH); 1 §, Nicosie (SDEI).

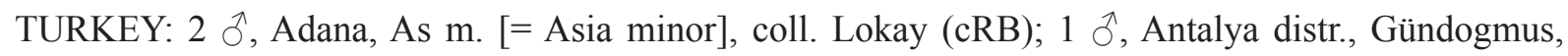
27 Sep.1980, leg. Lodos (NMBE). 


\section{Remarks}

The type locality given in the original description "Auf Rhodos" [on Rhodes] is wrong and should not be used in faunistic references, as in Dalla Torre et al. (1936). Charles Zürcher himself (Zürcher 1919: 159) corrected the origin of the type specimens from "Rhodes" to "L' île de Chypre". The present record from Gündogmus is the first for mainland Turkey.

\section{Ecology}

Only the given data; collected late May and in September.

Polydrusus (Conocetus) stierlini (Schilsky, 1910)

Figs 1E-F, 5C, 7C, 9E, 12

\section{Material examined}

Type material

Two syntype specimens:

GREECE: 1 + , Stierlini * Schils. // D. Leonh. // SYNTYPUS Polydrosus [sic!] stierlini Schilsky, 1910 labelled by MNHUB 2014 [printed on red paper] (MFN); 1 \%, Attica // coll. Stierlin [printed] // Stierlini m. [mihi] // Syntypus [printed on red paper] (SDEI).

\section{Type locality}

GREECE: Attica.

\section{Other material examined}

GREECE: 1 + , Erythrae, 12 Jun. [19]86, Porto Jermane, Attiki, leg. N. Neubauer (SDEI); 1 , RhodosOstküste, 1 Apr.-13 Apr. 1991, leg. H.-J. Grunwald (cHW); 1 ㅇ, Peloponnes, Taigetos, Anavriti, 1100-

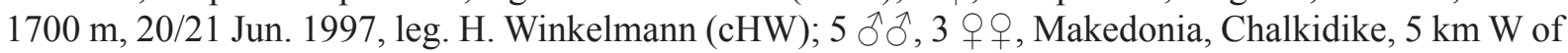

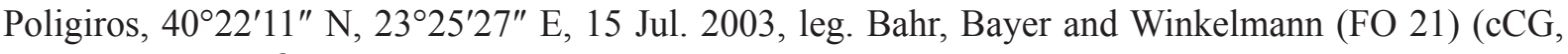

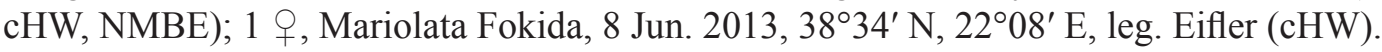

TURKEY: 1 \&, Tu Gyndogans [= Gündogan], 600 m, leg. S. Lundberg (SDEI); 1 q, Tu O. Telar... [illegible], 100 m, leg. S. Lundberg (SDEI); 1 क, Sarigin [= Sarigerme?], leg. S. Lundberg (SDEI); 1 ㅇ, Smyrna, coll. Apfelbeck (NMB); 1 , N. Termessos, 1 Jun. 1989, leg. G. Gillerfors (SDEI); 1 ㅇ, Türkei, leg. W. Wittmer (NMB); 1 +, Myra, 5 May 1991, leg. B. Zbuzek (cRB); 1 q, Olüdeniz, 8 May 1991, Fethyie env., leg. B. Zbuzek (cRB); 3 + , Prov. Hatay, (Hatay S) Sungur, 800 m, 29 Jul. 1998, leg. H. Winkelmann (cHW); 1 q Antakya, Samandag, Seleukeia Pieria, 40 m, 1 Jun. 2002, leg. S. Zoia and C. Giusto, coll. L. Magnano (BMNH).

\section{Variability}

The typical robust head (Fig. 5C) is more pronounced in females than in males, which are generally more slender (Fig. 1E-F). Interestingly, among the few specimens examined, males were much more rarely collected than females.

\section{Ecology}

Collected from April to July, from sea level to montane (exceptionally subalpine) altitudes. 


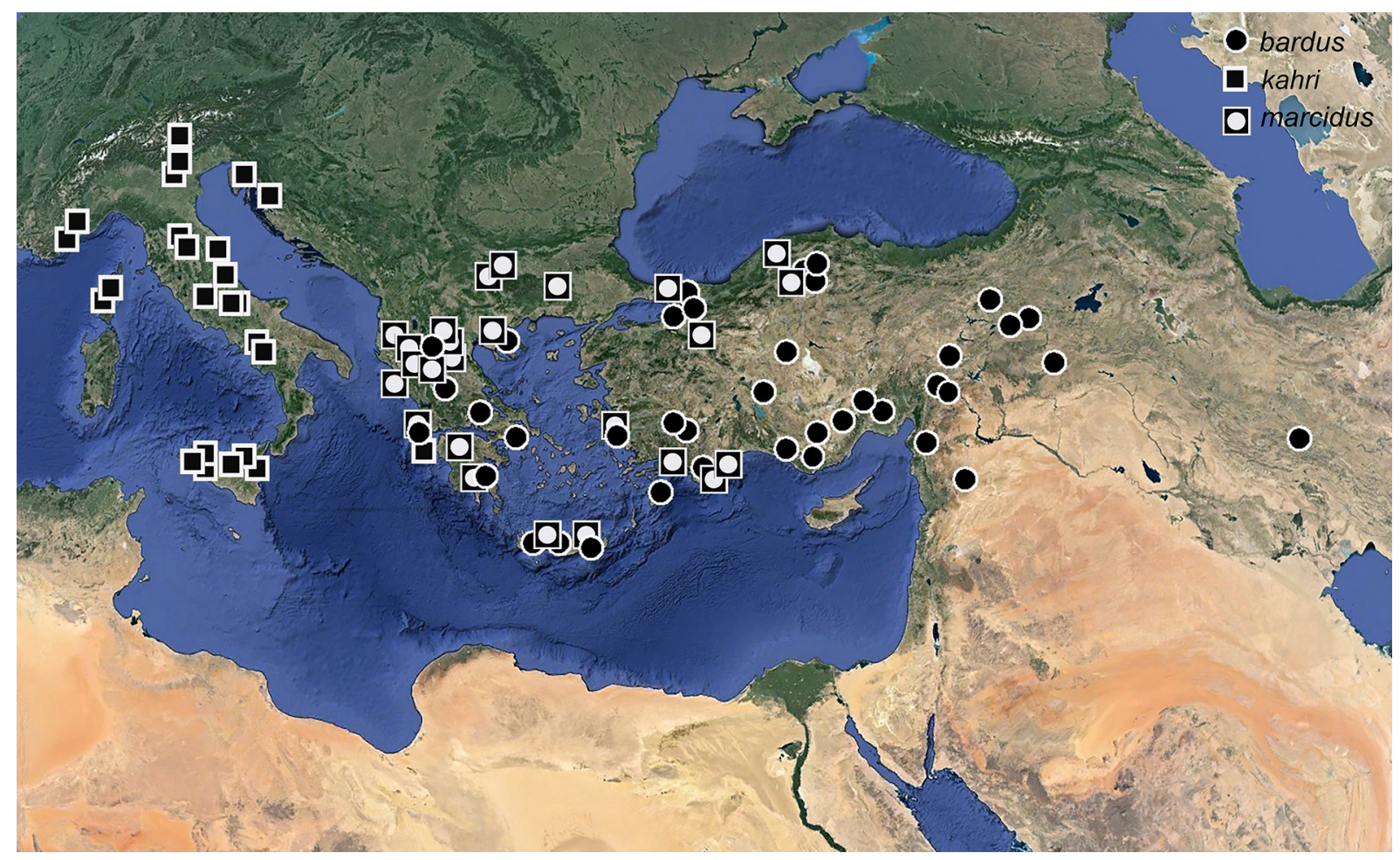

Fig. 10. Map showing records of Polydrusus (Denticonocetus) kahri Kirsch, 1865, and two species of $P$. (Conocetus). Those of P. kahri for France were taken from Hoffmann (1950), whereas literature records from Bosnia-Hercegovina, Macedonia and Montenegro, not verified by specimens examined, were not included (map copyright: Google).

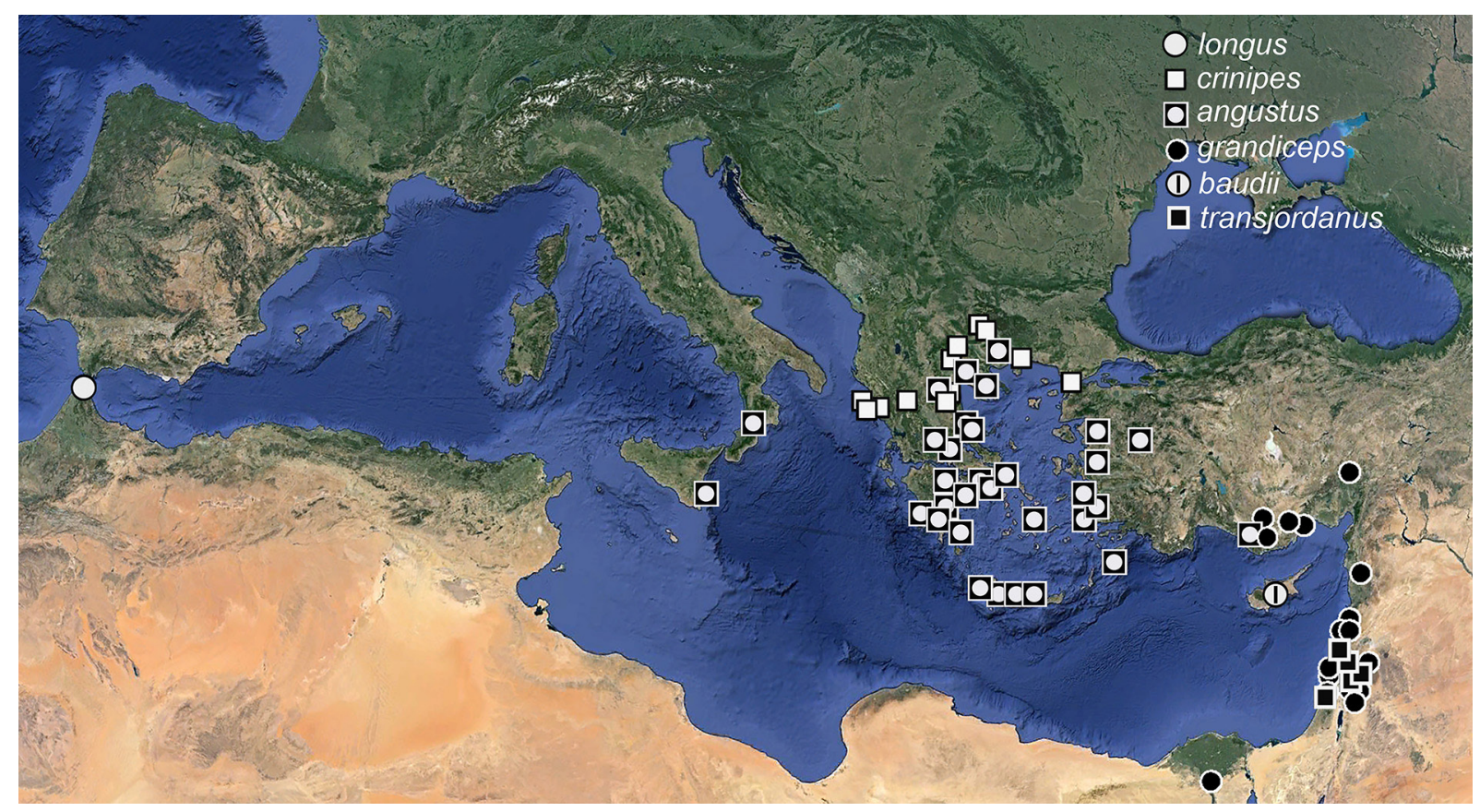

Fig. 11. Map showing records of five species of Polydrusus (Conocetus) and of P. (incertae sedis) longus (Stierlin, 1884) (map copyright: Google). 


\section{Incertae sedis}

Polydrusus longus (Stierlin, 1884)

Fig. 11

\section{Type locality}

MOROCCO: Tangiers.

\section{Remarks}

Unfortunately no specimen of this seemingly very characteristic species, based on its narrow and very elongate elytra (length: $5 \mathrm{~mm}$, width: $1 \mathrm{~mm}$ ), which are 3 times as long as wide (Stierlin 1884), could be examined in the present review. Stierlin (1884) compared P. longus with P. bardus (whereas with present knowledge, $P$. crinipes sp. nov. was meant). However, the distribution of $P$. longus, which seems to be restricted to the westernmost part of the Mediterranean basin, in comparison with the eastern focus of all other species of the subgenus, is at least surprising. One specimen from the BMNH was determined by L. Magnano as "Polydrusus (Conocetus) longus Stl.", but belongs instead to Polydrusus (Chomatocryptus) xanthopus Gyllenhal, 1834. As the placement in Conocetus cannot be verified, and is doubtful based on the deviant distribution and the very elongate habitus, the species is preliminarily treated as incertae sedis.

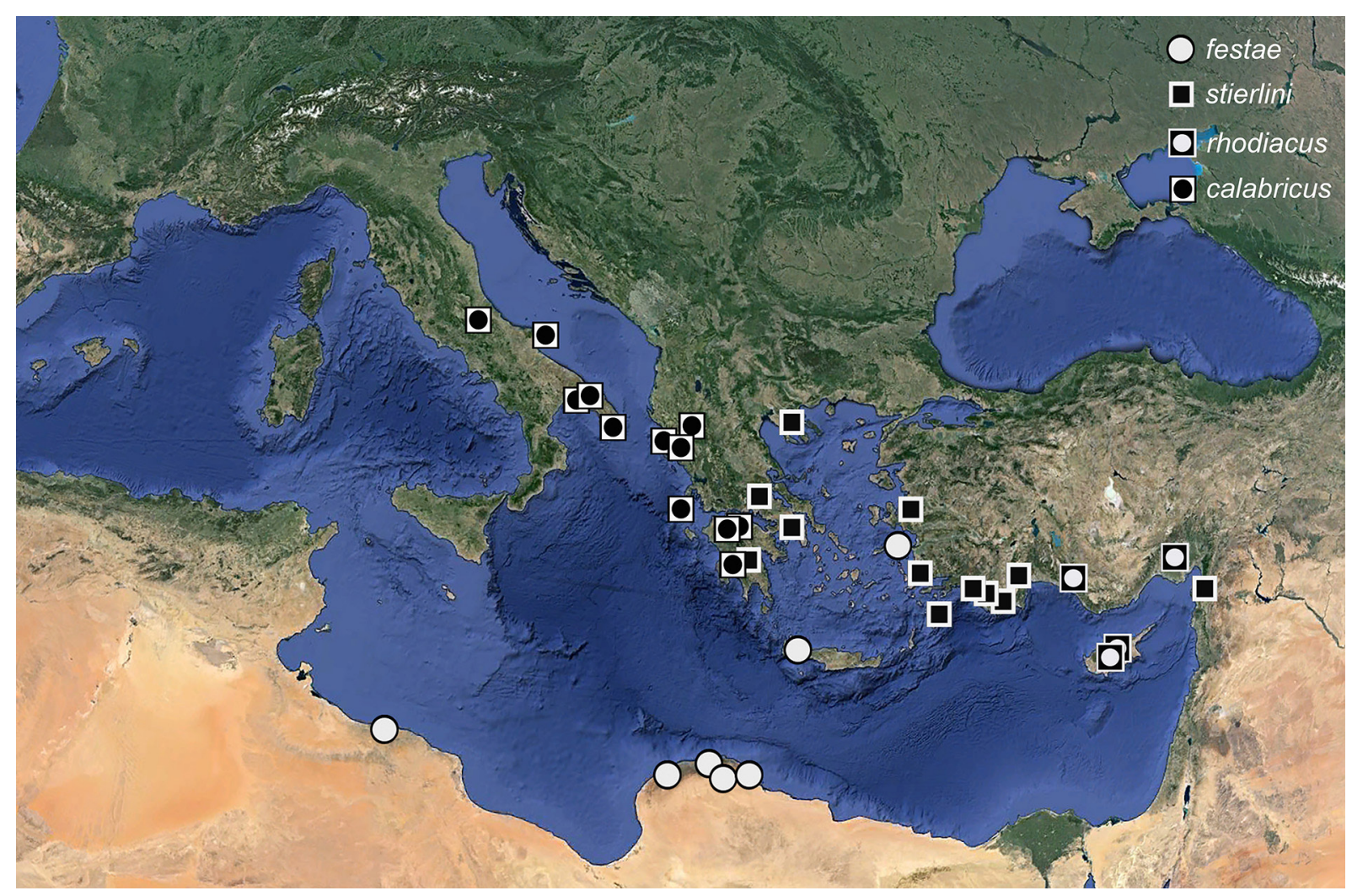

Fig. 12. Map showing records of four species of Polydrusus (Conocetus); additional records of P. festae (F. Solari, 1925) are taken from Solari (1925) (map copyright: Google). 


\section{Key to the species of Polydrusus (Conocetus) and P. (Denticonocetus) subgen. nov.}

1. All femora toothed (Fig. 1A-B) ..................... P. (Denticonocetus subgen. nov.) kahri Kirsch, 1865

- Femorae untoothed (Conocetus) 2

2. Elytra oval, shoulders not pronounced (brachypterous; Fig. 1C-D); scutellum with densely standing scales; eyes big and convex P. festae (F. Solari, 1925)

- Elytra long oval or parallel sided (males) or parallel at least at basal half, then widened towards apex (females), shoulders pronounced (hind wings present; not verified in P. longus); scutellum with inconspicuous vestiture; eyes of various shape....

3. Elytra 3 times as long as width of both elytra P. (incertae sedis) longus (Stierlin, 1884)

- Elytra less than 3 times as long as width of both elytra

4. Legs with either whitish hairs or hairs with green lustre, no scales ............................................. 5

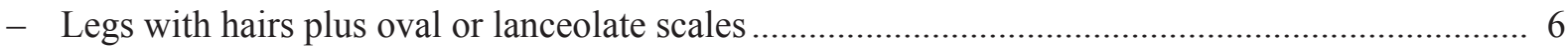

5. Legs reddish-brown with very long, raised, white hairs with a pearl-like lustre. Eyes less bulged, long oval drop-shaped as in P. angustus (Figs 2E-F, 3C-D).

P. rhodiacus (Schilsky, 1912)

- Legs bright reddish-brown to yellowish, with shorter hairs with green lustre. Eyes oval, clearly bulged (Fig. 3A-B)

P. crinipes sp. nov.

6. Head very robust (especially in females); eyes remarkably small, placed laterally, oval and bulging; temples mostly longer than diameter of an eye. Frons and epifrons bulged, therefore head and rostrum appear swollen (Figs 1E-F, 5C). P. stierlini (Schilsky, 1910)

- Head different

7. Bigger and long-oval, elongate species with apex of elytra elongately pointed (Fig. 3C-F); scales oval, dense, standing, with blue-greenish sheen; femora robust; body colour dark-brown to blackish; eyes either conspicuously long-oval (Fig. 5D) or circular and bulging ?

Smaller species with parallel sided elytra, apex of elytra not elongately pointed; scales brighter golden-greenish or other (coppery, brownish, or whitish to pearl-like); body colour brighter; eyes oval but not long-oval......

8. Eyes conspicuously long-oval drop shaped and less bulged (Figs 3C-D, 5D)

- Eyes circular and bulged (Fig. 3E-F)

P. angustus (Lucas, 1854)

P. calabricus (Faust, 1890)

9. Hairs on head, pronotum and elytra whitish, conspicuous, long and semi-raised (at least $45^{\circ}$ angle). Body colour mostly well visible through rather scarce, often spotty standing, oval to lanceolate whitish scales with a greenish pearl-like lustre; scales on sides of pronotum mostly denser standing ....... 10

- Hairs less conspicuous, shorter and more adjacent; scales on elytra and pronotum different......... 11

10. Eyes big (Fig. 5A), protruding (note: males have bigger eyes than females); body mostly dark brown; scales generally narrower, lanceolate-oval, sparsely standing (often spotty), with whitish to pearl-like lustre (only exceptionally slightly greenish); penis more robust, tip longer, truncated (Fig. 6A-D) .............................................................. P. grandiceps (Desbrochers des Loges, 1875)

- Eyes small (Fig. 5B), less protruding (note: males have bigger eyes than females); body colour brighter brown; scales generally more rounded-oval, denser and more regular standing with greenish pearl lustre; penis more gracile, tip shorter, slightly rounded (Fig. 6E-H) ... 
11. Scales on upper side of body vivid green, circular, dense and regularly standing, hairs in-between short and adjacent (Fig. 4A-B).

P. bardus Gyllenhal, 1834

- Scales on elytra generally circular, but with lanceolate ones intermixed, therefore appearing spotty or cloudy (except males of $P$. baudii, see below), hairs in-between longer. Colour varying from greenish to brownish, more lanceolate scales around disc of pronotum (thus disc appearing bare).

12. Males.

- Females (the females of the two following species are very similar, and thus difficult to distinguish)

13. Males more robust, broad, laterally and dorsally bulged. Elytra broader. Vestiture cloudy, with lanceolate scales intermixed (Fig. 4C-D). Penis robust (Fig. 8C), internal sac with long and slender sclerite (Fig 9M); males only known from Turkey, possible parthenogenetic reproduction elsewhere (= geographical parthenogenesis)

P. marcidus Kiesenwetter, 1864

- Males more gracile and of about the same size as females; narrower, weakly rounded laterally and hardly bulged dorsally. Elytra long-oval, parallel along middle, pointed towards apex. Vestiture with regular-standing circular scales (Fig. 4E-F). Penis gracile (Fig. 8D), internal sac with short and stout sclerite (Fig. 9N). Known from Cyprus only

P. baudii (Faust, 1889)

14. Elytral hairs brighter, mostly whitish (Fig. 4D)

P. marcidus Kiesenwetter, 1864

- Elytral hairs mostly (but not always) darker (Fig. 4F). Known from Cyprus only

P. baudii (Faust, 1889)

\section{Discussion}

In this review, the often-misinterpreted species of the subgenus Conocetus are revised, and the use of synonymous names is clarified through the study of type specimens and topotypical material, resulting in seven new synonymies.

Based on its morphology, Polydrusus kahri does not belong in Conocetus, nor in any other established subgenus. To accommodate this species, we herein propose the new subgenus Denticonocetus.

The re-evaluation of the morphology of Conocetus revealed that the following characters are important for specific determination: i) body shape, ii) shape of head, iii) shape of eyes, iv) vestiture of body (including length and orientation of hairs), v) vestiture of legs, vi) shape of scales, vii) male genitalia including internal sac. Using these characters, two new species are described: Polydrusus (Conocetus) transjordanus sp. nov. and Polydrusus (Conocetus) crinipes sp. nov..

Body size, shape of pronotum, colour of scales, colour of legs, often body colour, and female genital organs are intraspecifically variable, and thus may be misleading. The most surprising faunistic results are new records from Italy with $P$. angustus, Greece with the apterous $P$. festae, extending its distribution across the Mediterranean Sea, and records of $P$. rhodiacus from mainland Turkey, showing that the species is not endemic to Cyprus.

\section{Acknowledgements}

Many thanks for considerable support to Martin Albrecht (Bolligen, Switzerland), Friedhelm Bahr (Viersen, Germany), Max Barclay (BMNH), Christoph Bayer (Berlin, Germany), Lutz Behne (SDEI), Johannes Bergsten (NRM), Piotr Białooki (State Plant Health and Seed Inspection, Gdańsk, Poland), Matthias Borer (NMB), Roman Borovec (Smidary, Czech Republic), Carlo Braunert (Mendsorf, Luxembourg), Manfred Egger (Innsbruck, Austria), Laibale Friedman (SMNHTAU), Michael Geiser (BMNH), Julien Haran (Montpellier, France), Thomas Kissling (Zürich, Switzerland), Jochen Messutat 
(Preußisch-Oldendorf, Germany), Christian Monnerat (Info fauna - CSCF, Neuchâtel), Gerd and Uschi Müller (Frechen, Germany), Hans Mühle (München, Germany), Jean Pelletier (Monnaie, France), Karel Schön (Litvínov, Czech Republic), Laurent Schott (La Chapelle Saint Luc, France), Eva Sprecher (NMB), Joachim Willers (MFN), Herbert Winkelmann (Berlin, Germany) and Wolfgang Ziegler (Rondeshagen, Germany). I am grateful to the referees for their helpful comments on and corrections to the manuscript.

\section{References}

Abbazzi P. \& Maggini L. 2009. Elenco sistematico-faunistico dei Curculionoidea italiani, Scolytidae e Platypodidae esclusi (Insecta, Coleoptera). Aldrovandia 5: 29-216.

Alonso-Zarazaga M.A., Barrios H., Borovec R., Bouchard P., Caldara R., Colonnelli E., Gültekin L., Hlaváć P., Korotyaev B., Lyal C.H.C., Machado A., Meregalli M., Pierotti H., Ren L., Sánchez-Ruiz M., Sforzi A., Silfverberg H., Skuhrovec J., Trýzna M., Velázquez de Castro A.J. \& Yunakov N.N. 2017. Cooperative Catalogue of Palaearctic Coleoptera Curculionoidea. Monografías electrónicas S.E.A. 8, Sociedad Entomológica Aragonesa S.E.A., Zaragoza.

Bertolini S. 1868. Neue Käferarten des Trentinogebietes. Verhandlungen der Kaiserlich-Königlichen Zoologisch-Botanischen Gesellschaft in Wien 18: 119-120.

Chevrolat L.A.A. 1869. Description de 14 nouvelles espèces de curculionides d'Europe, d'Algérie, de l'Asie Mineure et d'un nouveau genre de cette famille. Coleopterologische Hefte 5: 71-77.

Colonnelli E. 2003. A revised checklist of Italian Curculionoidea (Coleoptera). Zootaxa 337: 1-142.

Dalla Torre K.W., Emden M. van \& Emden F. van. 1936. Curculionidae: Brachyderinae. I. Pars 147. In: Schenkling S. (ed.) Coleopterorum Catalogus: auspiciis et auxilio: 132. Dr W. Junk, s'-Gravenhage.

Desbrochers des Loges J. 1872. Diagnoses d'espèces nouvelles de Coléoptères appartenant aux genres Polydrusus, Thylacites, Tanymecus, Scythropus, Metallites et Phaenognathus. Annales de la Société entomologique de France 5 (1) [1871]: 231-246.

Desbrochers des Loges J. 1875. Diagnoses de Curculionides inédits. Opuscules Entomologiques (Coléoptères) 1: 1-36.

Desbrochers des Loges J. 1900. Espèces inédites de curculionides de l'Ancien Monde. VI [sic!]. Le Frelon 8 (5): 1-16.

Desbrochers des Loges J. 1902. Révision des curculionides appartenant aux genres Eudipnus et Conocoetus et genres voisins et au groupe des Scythropidae, suivie de rectifications synonymiques observations diverses. Le Frelon 10 [1901-1902]: 113-136.

Desbrochers des Loges J. 1903. Description d'un Polydrosus nouveau de Corse. Le Frelon 11 (8): 114-115.

Faust J. 1889. Berichte über die von E. v. Oertzen im Jahre 1887 in Griechenland u. Klein-Asien gesammelten Coleopteren. VII. Griechische Curculioniden. Besprechung und Beschreibung einiger Arten. Deutsche Entomologische Zeitschrift 1889: 66-98.

Faust J. 1890. Rüsselkäfer aus den Mittelmeer-Ländern. Deutsche Entomologische Zeitschrift 1890 (2): 321-336.

Germann C., Müller G., Müller U. \& Schön K. 2015. An annotated checklist of the weevil fauna of Samos Island with new records for Greece (Coleoptera, Curculionoidea). Contributions to Natural History 27: 1-26.

Hoffmann A. 1950. Coléoptères Curculionides. Première partie. Faune de France 52, Editions Paul Lechevalier, Paris. 
Horn W., Kahle I., Friese G. \& Gaedike R. 1990. Collectiones Entomologicae: eine Kompendium über den Verbleib entomologischer Sammlungen der Welt bis 1960. Vols 1-2. Akademie der Landwirtschaftswissenschaften der Deutschen Demokratischen Republik, Berlin.

Kiesenwetter H. von 1864. Beitrag zur Käferfauna Griechenlands. Neuntes Stück. Curculionidae. Berliner Entomologische Zeitschrift 8: 239-293.

Kirsch T.F.W. 1865. Kleinere Mittheilungen. Berliner Entomologische Zeitschrift 9: 121-124.

Lucas P.H. 1854. Essai sur les animaux articulés qui inhabitent l'île de Crête. Revue et Magasin de Zoologie pure et appliquée (2) 6: 28-44.

Magnano L. 1964. Ricerche sulla fauna appenninica. LXXVII. Coleotteri curculionidi dell'Aspromonte. (X contributo alla conoscenza dei curculionidi). Memorie del Museo civico di storia naturale di Verona 12: $107-147$.

Schilsky J. 1910. Die Käfer Europas. Nach der Natur beschrieben von Dr. H.C. Küster und Dr. G Kraatz. Heft 46. Bauer und Raspe, Nuremburg.

Schilsky J. 1912. Die Käfer Europas. Nach der Natur beschrieben von Dr. H.C. Küster und Dr. G. Kraatz. Heft 48. Bauer und Raspe, Nuremburg.

Schoenherr C.J., Boheman C.H \& Gyllenhal L. Genera et species Curculionidum, cum synonymia hujus familiae. Species novae aut hactenus minus cognitae, descriptionibus a Dom. Leonardo Gyllenhal, C. H. Boheman, et entomologis aliis illustratae. Tomus secundus. - Pars prima. Roret, Paris.

https://doi.org/10.5962/bhl.title.8952

Schott L. 2014. Polydrusini 707-709. In: Tronquet M. (ed.) Catalogue des Coléoptères de France. Association Roussillonnaise d'Entomologie, Perpignan.

Solari F. 1925. Missione zoologica del Dr. E. Festa in Cirenaica. XIV. Coleotteri. In: Dodero A. (ed.) Bolletino dei Musei di zoologia ed anatomia comparata della Università di Torino (nueva serie) 39 (23): $1-31$.

Stierlin W.G. 1884. Bestimmungs-Tabellen europäischer Rüsselkäfer. II. Brachyderidae. Mittheilungen der Schweizerischen Entomologischen Gesellschaft 7: 57-98. https://doi.org/10.5169/seals-400449

Stierlin W.G. 1888a. Beschreibung einiger neuen Käferarten. Mittheilungen der Schweizerischen Entomologischen Gesellschaft 8 (2): 48-61. https://doi.org/10.5169/seals-400479

Stierlin W.G. 1888b. Beschreibung einiger neuer europäischen Rüsselkäfer. Mittheilungen der Schweizerischen Entomologischen Gesellschaft 8 [1888-1893]: 9-20. https://doi.org/10.5169/seals-400508

Yunakov N. 2013. Polydrusini. In: Löbl I. \& Smetana A. (eds) Catalogue of Palaearctic Coleoptera. Vol. 8: Curculionoidea II: 364-375. Brill, Leiden.

Zürcher C. 1919. Observations diverses. La vraie provenance du Polydrosus rhodiacus Schilsky (Coleoptera Curculionidae). Bulletin de la Société entomologique de France 9: 159.

Manuscript received: 14 September 2016

Manuscript accepted: 16 June 2017

Published on: 5 January 2018

Topic editor: Gavin Broad

Section editor: Max Barclay

Desk editor: Chloe Chester 
Printed versions of all papers are also deposited in the libraries of the institutes that are members of the EJT consortium: Muséum national d'Histoire naturelle, Paris, France; Botanic Garden Meise, Belgium; Royal Museum for Central Africa, Tervuren, Belgium; Natural History Museum, London, United Kingdom; Royal Belgian Institute of Natural Sciences, Brussels, Belgium; Natural History Museum of Denmark, Copenhagen, Denmark; Naturalis Biodiversity Center, Leiden, the Netherlands; Museo Nacional de Ciencias Naturales-CSIC, Madrid, Spain; Real Jardín Botánico de Madrid CSIC, Spain. 